\title{
Resampling and ensemble techniques for improving ANN-based high-flow forecast accuracy
}

\author{
Everett Snieder, Karen Abogadil, and Usman T. Khan \\ Department of Civil Engineering, York University, 4700 Keele St, Toronto ON, M3J 1P3, Canada
}

Correspondence: Usman T. Khan (usman.khan@1assonde.yorku.ca)

Received: 21 August 2020 - Discussion started: 9 October 2020

Revised: 19 February 2021 - Accepted: 6 April 2021 - Published: 18 May 2021

\begin{abstract}
Data-driven flow-forecasting models, such as artificial neural networks (ANNs), are increasingly featured in research for their potential use in operational riverine flood warning systems. However, the distributions of observed flow data are imbalanced, resulting in poor prediction accuracy on high flows in terms of both amplitude and timing error. Resampling and ensemble techniques have been shown to improve model performance on imbalanced datasets. However, the efficacy of these methods (individually or combined) has not been explicitly evaluated for improving high-flow forecasts. In this research, we systematically evaluate and compare three resampling methods, random undersampling (RUS), random oversampling (ROS), and the synthetic minority oversampling technique for regression (SMOTER), and four ensemble techniques, randomised weights and biases, bagging, adaptive boosting (AdaBoost), and least-squares boosting (LSBoost), on their ability to improve high stage prediction accuracy using ANNs. These methods are implemented both independently and in combined hybrid techniques, where the resampling methods are embedded within the ensemble methods. This systematic approach for embedding resampling methods is a novel contribution. This research presents the first analysis of the effects of combining these methods on high stage prediction accuracy. Data from two Canadian watersheds (the Bow River in Alberta and the Don River in Ontario), representing distinct hydrological systems, are used as the basis for the comparison of the methods. The models are evaluated on overall performance and on typical and high stage subsets. The results of this research indicate that resampling produces marginal improvements to high stage prediction accuracy, whereas ensemble methods produce more substantial improvements, with or without resampling. Many of the
\end{abstract}

techniques used produced an asymmetric trade-off between typical and high stage performance; reduction of high stage error resulted in disproportionately larger error on a typical stage. The methods proposed in this study highlight the diversity-in-learning concept and help support future studies on adapting ensemble algorithms for resampling. This research contains many of the first instances of such methods for flow forecasting and, moreover, their efficacy in addressing the imbalance problem and heteroscedasticity, which are commonly observed in high-flow and flood-forecasting models.

\section{Introduction}

Data-driven models such as artificial neural networks (ANNs) have been widely and successfully used over the last 3 decades for hydrological forecasting applications (Govindaraju, 2000; Abrahart et al., 2012; Dawson and Wilby, 2001). However, some studies have noted that these models can exhibit poor performance during high-flow (or stage) hydrological events (Sudheer et al., 2003; Abrahart et al., 2007; de Vos and Rientjes, 2009), with poor performance manifesting as late predictions (i.e. timing error), underpredictions, or both. For flow-forecasting applications such as riverine flood warning systems, the accuracy of high stage predictions is more important than that of a typical stage. One cause of poor model accuracy on a high stage is the scarcity of representative sample observations available with which to train such models (Moniz et al., 2017a). This is because stage data typically exhibit a strong positive skew, referred to as an imbalanced domain; thus, there may only be a small number of flood observations within decades of samples. Consequently, 
objective functions that are traditionally used for training ANNs (e.g. mean squared error, MSE, sum of squared error SSE), which equally consider all samples, are biased towards values that occur most frequently and are reflected by poor model performance on high-flow or stage observations (Pisa et al., 2019). Sudheer et al. (2003) also point out that such objective functions are not optimal for non-normally distributed data. This problem is exacerbated when such metrics are also used to assess model performance; regrettably, such metrics are the most widely used in water resource applications (Maier et al., 2010). As a result, studies that assess models using traditional performance metrics risk overlooking deficiencies in high stage performance.

Real-time data-driven flow-forecasting models frequently use antecedent input variables (also referred to as autoregressive inputs) for predictions. Several studies have attributed poor model prediction on high stage to model overreliance on antecedent variables (Snieder et al., 2020; Abrahart et al., 2007; de Vos and Rientjes, 2009; Tongal and Booij, 2018). Consequently, the model predictions are similar to the most recent antecedent conditions, sometimes described as a lagged prediction (Tongal and Booij, 2018). In other words, the real-time observed stage at the target gauge is used as the predicted value for a given lead time. This issue is closely linked to the imbalanced domain problem as frequently occurring stage values typically exhibit low temporal variability compared to infrequent, high stage values; this phenomenon is further described in Sect. 2.

Improving the accuracy of high stage or flow forecasts has been the focus of many studies. Several studies have examined the use of preprocessing techniques to improve model performance. Sudheer et al. (2003) propose using a WilsonHilferty transformation to change the skewed distribution of stage data. The study found that transforming the target data reduces annual peak flow error produced by ANN-based daily flow-forecasting models. Wang et al. (2006) evaluate three strategies for categorising streamflow samples, based on a fixed-value flow threshold, unsupervised clustering, and periodicity; separate ANN models are trained to predict each flow category and combined to form a final prediction. The periodicity-based ANN, which detects periodicity from the autocorrelation function of the target variable, is found to perform the best out of the three schemes considered. Fleming et al. (2015) address the issue of poor high-flow performance by isolating a subset of daily high flows by thresholding based on a fixed value. By doing so, traditional objective functions (e.g. MSE) become less influenced by the imbalance of the training dataset. ANN-based ensembles trained on high flows are found to perform well, though the improvements to high-flow accuracy are not directly quantified, as the high-flow ensemble is not compared directly to a counterpart trained using the full training dataset.

An alternative approach to improving high-flow forecast accuracy has been to characterise model error as having amplitude and temporal components (Seibert et al., 2016). Abra- hart et al. (2007) use a specialised learning technique in which models are optimised based on a combination of root mean square error (RMSE) and a timing error correction factor, which is found to improve model timing for short lead times but has little impact on higher lead times. de Vos and Rientjes (2009) use a similar approach, in which models that exhibit a timing error are penalised during calibration. The technique is found to generally reduce timing error at the expense of amplitude error.

Finally, there is considerable evidence that ensemblebased and resampling techniques improve prediction accuracy of infrequent samples (Galar et al., 2012). Ensemble methods, such as bootstrap aggregating (bagging) and boosting, are known for their ability to improve model generalisation. Such methods are widely used in classification studies and are increasingly being adapted for regression tasks (Moniz et al., 2017b). However, ensemble methods alone do not directly address the imbalance problem, as they typically do not explicitly consider the distribution of the target dataset. Thus, ensemble methods are often combined with preprocessing strategies to address the imbalance problem (Galar et al., 2012). Resampling, which is typically used as a preprocessing method, can be used to create more uniformly distributed target datasets or generate synthetic data with which to train models (Moniz et al., 2017a). Resampling also promotes diversity in learning when embedded in ensemble algorithms (rather than when used as a preprocessing strategy). Examples of such combinations appear in the machinelearning literature but are typically developed for ad hoc applications (Galar et al., 2012).

However, the efficacy of these methods (a combination of resampling strategies with ensemble methods) has not been systematically investigated for flow-forecasting applications. While previous studies have provided comparisons of ensemble methods, none have explicitly studied their effects on high-flow prediction accuracy, which has only received little attention within the context of the imbalance problem in general. Additionally, previous research uses resampling as a preprocessing technique, whereas in this research, resampling is embedded within the ensembles to promote diversity in learning. Thus, the main objective of this research is to develop a systematised framework for combining several different resampling and ensemble techniques with the aim of improving high-flow forecasts using ANNs. Three resampling techniques, random undersampling (RUS), random oversampling (ROS), and the synthetic minority oversampling technique for regression (SMOTER), and four ensemble algorithms, randomised weights and biases (RWB), bagging, adaptive boosting for regression (AdaBoost), and least-squares boosting (LSBoost), will be investigated to address the issues related to high-flow forecasts, i.e. the imbalanced domain problem and heteroscedasticity. Each combination of these methods will be explicitly evaluated on their ability to improve model performance on high stage (infrequent) data subsets along with the typical (frequent) data 
subsets. Such a framework and comparison, to address the imbalanced domain, have not been presented in the existing literature. Lastly, while only selected resampling and ensemble techniques are presented, many of which are the first instances of their use for high-flow forecasting, this proposed framework may easily be expanded to resampling and ensemble strategies beyond those included in this research.

The remainder of the paper is organised as follows: first, in Sect. 2 we present the baseline ANN flow forecast models, which are used as the individual learners for the ensembles, for two Canadian watersheds, followed by a performance analysis of these models to highlight the imbalance domain problem and illustrate the heteroscedasticity of baseline model residuals. The two watersheds, with differing hydrological characteristics but both prone to riverine floods, are the Bow River watershed (in Alberta) and the Don River watershed (in Ontario). Section 3 provides a review and applications of each resampling method and ensemble technique followed by a description of the implementation of each approach in this research and model evaluation method. Lastly, Sect. 4 includes the results and discussion from the two case studies.

\section{Early investigations}

The following section provides descriptions for the two watersheds under study. The parametrisation of the single ANN models to predict the stage in each watershed (referred to as the individual learners) is described. The outputs of the individual learners are used to exemplify the inability of these ANNs to accurately predict high stage (from both an amplitude and temporal error perspective) and to illustrate the imbalance problem.

\subsection{Study area}

The Bow and Don rivers are featured as case studies in this research to evaluate methods for improving the accuracy of high stage data-driven forecasts. The Bow River, illustrated in Fig. 1a, begins in the Canadian Rockies mountain range and flows eastward through the city of Calgary, where it is joined by the Elbow River. The Bow River's flow regime is dominated by glacial and snowmelt processes which produce annual seasonality. The Bow River watershed has an area of approximately $7700 \mathrm{~km}^{2}$ upstream of the target stage monitoring station in Calgary and consists of predominantly natural and agricultural land cover. The city of Calgary has experienced several major floods (recently in 2005 and 2013), and improvements to flow-forecasting models have been identified as a key strategy for mitigating flood damage Khan et al. (2018).

The Don River, illustrated in Fig. 1b, begins in the Oak Ridges Moraine and winds through the Greater Toronto Area until it meets Lake Ontario in downtown Toronto. The

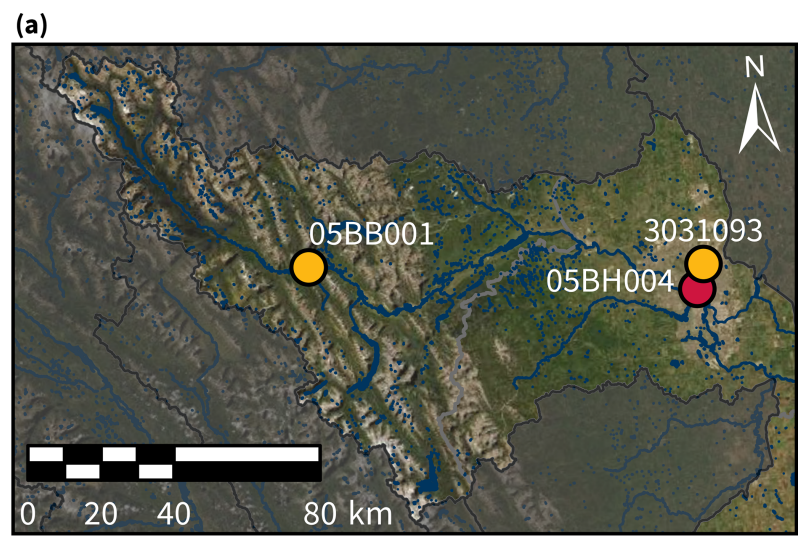

(b)

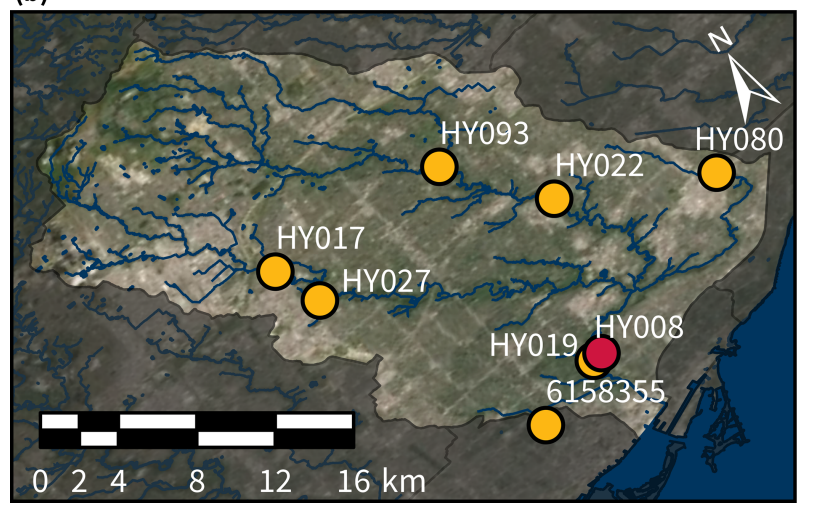

Figure 1. Bow (a) and Don (b) River basins upstream of Calgary and Toronto, respectively. Surface watercourses and waterbodies are shown in blue. The target stage monitoring stations are red, while upstream hydrometeorological monitoring stations (stage, precipitation, and temperature) are yellow. Aerial imagery obtained from (C) Esri (Esri, 2020). Surface water and watershed boundaries obtained from (C) Scholars GeoPortal (DMTI Spatial Inc., 2014a, b, c, 2019) and the (C) TRCA (Toronto and Region Conservation Authority, 2020b).

$360 \mathrm{~km}^{2}$ Don River watershed is heavily urbanised, which results in the high stage seen in the River being attributable to the direct runoff following intense rainfall events. Its urbanised landscape has also contributed to periodic historical flooding (Toronto and Region Conservation Authority, 2020a). Persistent severe flooding (recently in 2005 and 2013) has motivated calls for further mitigation strategies such as improved flow forecast models and early warning systems (Nirupama et al., 2014).

Data from November to April and November to December were removed from the Bow and Don River datasets, prior to any analysis; these periods are associated with ice conditions. The histograms in Fig. 2 illustrate the imbalanced domains of the target stage for both rivers. A high stage threshold $\left(\Theta_{\mathrm{HS}}\right)$ is defined, which is used to distinguish between typical and high stage. Stage values greater than the threshold are referred to as high stage $\left(q_{\mathrm{HS}}\right)$ and stage values below the threshold as typical stage $\left(q_{\mathrm{TS}}\right)$. Target stage statistics for 
Table 1. Target variable statistics for the Bow and Don River watersheds.

\begin{tabular}{llrrrrr}
\hline River & Subset & $\begin{array}{r}\text { Mean } \\
(\mathrm{m})\end{array}$ & $\begin{array}{r}\text { Min. } \\
(\mathrm{m})\end{array}$ & $\begin{array}{r}\text { Max. } \\
(\mathrm{m})\end{array}$ & $\begin{array}{r}\text { Skew. } \\
(-)\end{array}$ & $\begin{array}{r}\text { Var. } \\
\left(\mathrm{m}^{2}\right)\end{array}$ \\
\hline \multirow{2}{*}{ Bow } & $q$ & 1.28 & 0.92 & 3.07 & 1.18 & 0.067 \\
& $q_{\mathrm{TS}}$ & 1.18 & 0.92 & 1.47 & 0.21 & 0.022 \\
& $q_{\mathrm{HS}}$ & 1.69 & 1.47 & 3.07 & 1.85 & 0.039 \\
\hline \multirow{2}{*}{ Don } & $q$ & 77.62 & 77.51 & 79.21 & 3.78 & 0.018 \\
& $q_{\mathrm{TS}}$ & 77.58 & 77.51 & 77.67 & 0.59 & 0.0017 \\
& $q_{\mathrm{HS}}$ & 77.82 & 77.68 & 79.21 & 2.99 & 0.034 \\
\hline
\end{tabular}

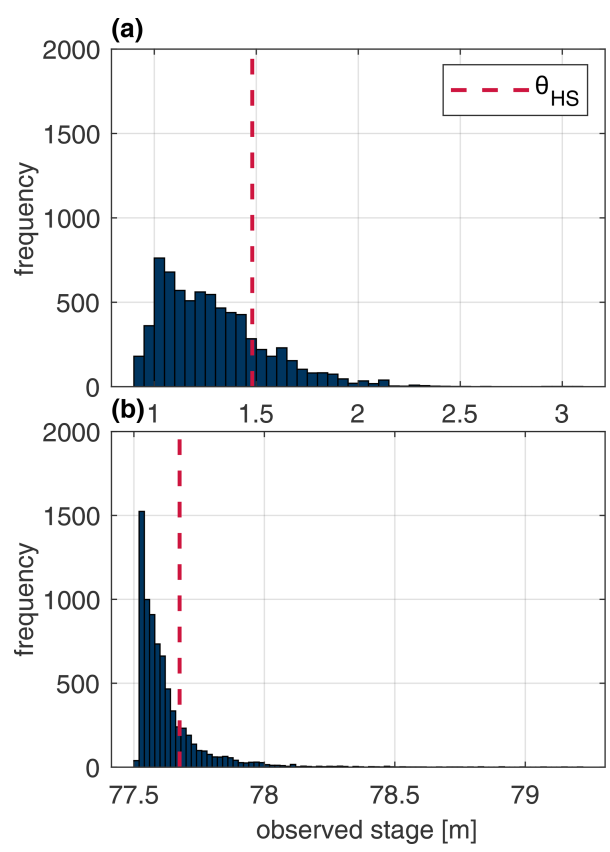

Figure 2. Histograms of the observed stage for the (a) Bow River $6 \mathrm{~h}$ stage and (b) Don River hourly stage. The dashed red line indicates the fixed threshold used to distinguish between typical and high stage values.

the Bow and Don rivers are provided for the complete stage distribution as well as the $q_{\mathrm{TS}}$ and $q_{\mathrm{HS}}$ subsets in Table 1 .

The use of a fixed threshold for distinguishing between common (frequent) and rare (infrequent) samples is used both in flow forecasting (Crochemore et al., 2015; Razali et al., 2020; Fleming et al., 2015) and in more general machine-learning studies that are focused on the imbalance problem (Moniz et al., 2017a). In this research, the high stage threshold is simply and arbitrarily taken as the 80th percentile value of the observed stage. The threshold value is ideally derived from the physical characteristics of the river (i.e. the stage at which water exceeds the bank or is associated with a specified return period); unfortunately this sitespecific information is not readily available for the subject watersheds. An important consideration to make while se- lecting a $\Theta_{\mathrm{HS}}$ value is that it produces a sufficient number of high stage samples; having too few samples risks overfitting and poor generalisation. The distinction between typical and high stage is used in some of the resampling techniques in Sect. 3.1 and for assessing model performance in Sect. 3.4.

\subsection{Individual learner description}

The individual learner (sometimes called the base model or base learner) for both systems uses upstream hydrometeorological inputs (stage, precipitation, and temperature) to predict the downstream stage (the target variable). The multi-layer perception (MLP) ANN is used as the individual learner for this study, and the selected model hyperparameters are summarised in Table 2. The MLP ANN was chosen as the individual learner because it is the most commonly used machine-learning architecture for predicting water resource variables in river systems (Maier et al., 2010). The individual learner can be used for discrete value prediction or as a member of an ensemble in which a collection of models is trained and combined to generate predictions. Each ANN has a hidden layer of 10 neurons; a grid search of different hidden layer sizes indicated that larger numbers of hidden neurons have little impact on the ANN performance. Thus, to prevent needlessly increasing model complexity, a small hidden layer is favoured. The number of training epochs is determined using early stopping (also called stop training), which is performed by dividing the calibration data into training and validation subsets; training data are used to tune the ANN weights and biases, whereas the validation performance is used to determine when to stop training (Anctil and Lauzon, 2004). For this study, the optimum number of epochs is assumed if the error on the validation set increases for five consecutive epochs. Early stopping is a common technique for achieving generalisation and preventing overfitting (Anctil and Lauzon, 2004). Of the available data for each watershed, $60 \%$ are used for training, $20 \%$ for validation, and $20 \%$ for testing (the independent dataset). $K$-fold crossvalidation (KFCV) is used to evaluate different continuous partitions of training and testing data and is explained in greater detail in Sect. 3.4.2. The Levenberg-Marquardt algorithm was used to train the individual learners because of its speed of convergence and reliability (Lauzon et al., 2006; Maier and Dandy, 2000; Tongal and Booij, 2018). The full set of input and target variables used for both catchments is summarised in Table 3. For both rivers, the input variables are used to forecast the target variable four time steps in advance; i.e. for the Bow River, the model forecasts $24 \mathrm{~h}$ in the future, whereas for the Don River, the model forecasts $4 \mathrm{~h}$ in the future. Some of the input variables used in the Bow River model, including the minimum, mean, and maximum statistics, are calculated by coarsening hourly data to a $6 \mathrm{~h}$ time step. Several lagged copies of each input variable are used, which is common practice for ANN-based hydrological forecasting models (Snieder et al., 2020; Abbot and Marohasy, 
Table 2. Individual learner ANN model description used for both watersheds.

\begin{tabular}{ll}
\hline Model class & Artificial neural network \\
Architecture & Multi-layer perceptron \\
IVS & Partial correlation \\
Hidden neurons & 10 \\
Activation function & Tanh (hidden layer), linear (output layer) \\
Training algorithm & Levenberg-Marquardt backpropagation \\
Stopping criteria & Validation dataset \\
\hline
\end{tabular}

2014; Fernando et al., 2009; Banjac et al., 2015). For example, to forecast $x_{t}$ by four time steps, $x_{t-4}, x_{t-5}, x_{t-6}$, etc., may be used as input variables, as these variables are recorded automatically, in real time.

The partial correlation (PC) input variable selection (IVS) algorithm is used to determine the most suitable inputs for each model from the larger candidate set (He et al., 2011; Sharma, 2000). Previous research for the Don and Bow rivers found that PC is generally capable of removing non-useful inputs in both systems, achieving reduced computational demand and improved model performance (Snieder et al., 2020). The simplicity and computational efficiency of the PC algorithm method make it an appealing IVS algorithm for this application. The 25 most useful inputs amongst all the candidates listed in Table 3, determined by the PC algorithm, are used in the models for each watershed. A complete list of selected inputs is shown in Appendix A.

The Bow and Don River individual learners produce coefficients of Nash-Sutcliffe efficiency (CEs) greater than 0.95 and 0.75 , respectively. These scores are widely considered by hydrologists to indicate good performance (Crochemore et al., 2015). However, closer investigation of the model performance reveals that high stage samples consistently exhibit considerable error. This is plainly visible when comparing the observed hydrographs with the individual learner predictions, as shown in Figs. 3 and 4, for the Bow and Don rivers, respectively. Plotting the individual learner residuals against the observed stage, as in Fig. 5a and b, illustrates how the variance of the residuals about the expected mean of 0 increases with the increasing stage magnitude; Fleming et al. (2015) also describe the heteroscedastic nature of flow prediction models. This region of high stage also exhibits amplitude errors in excess of $1 \mathrm{~m}$, casting doubt on the suitability of these models for flood-forecasting applications. In Fig. $5 \mathrm{~b}$ and $\mathrm{c}$ the normalised inverse frequency of each sample point is plotted against the stage gradient, illustrating how the most frequent stage values typically have a low gradient with respect to the forecast lead time, given by $\left(q_{t+L}-q_{t}\right) / L$. Note that the inverse frequency is determined using 100 histogram bins. Thus, when such a relationship exists, it is unsurprising that model output predictions are similar to the most recent autoregressive input variable. Previous work that analysed trained ANN models for both subject watersheds

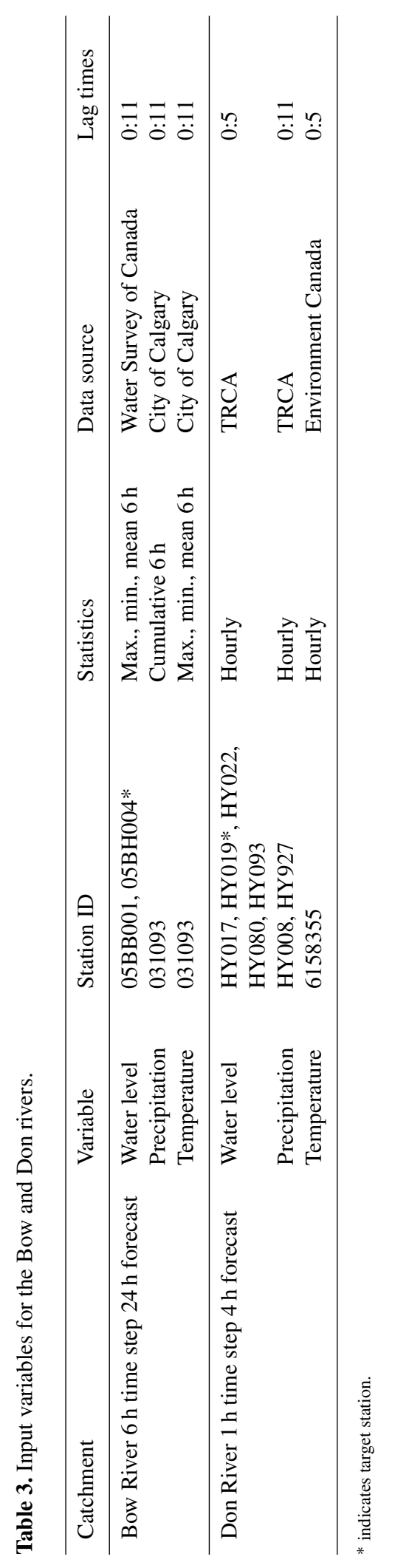

Hydrol. Earth Syst. Sci., 25, 2543-2566, 2021 
(a)

200120022003200420052006200720082009

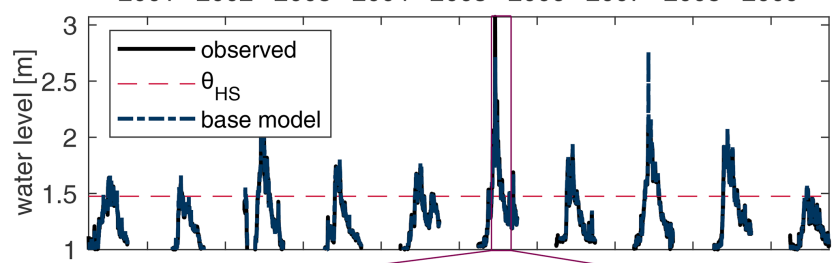

(b)

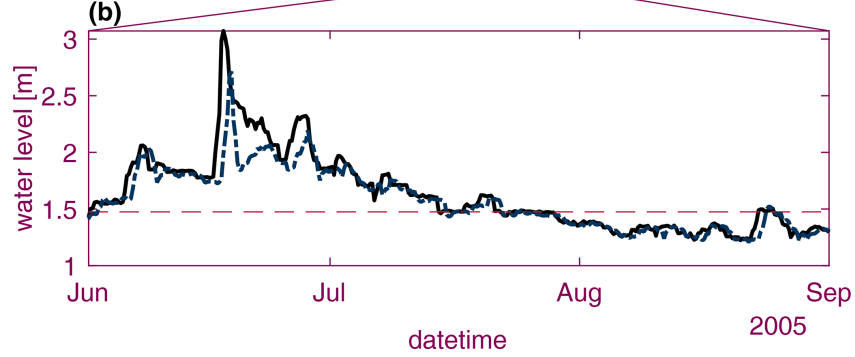

Figure 3. Observed and individual learner stage predictions for the Bow River system for all 10 years of an available stage (a) and a 3-month subset which contains a particularly high stage (b) to better distinguish between the two hydrographs. The dashed red line indicates the fixed threshold used to distinguish between typical and high stage values.

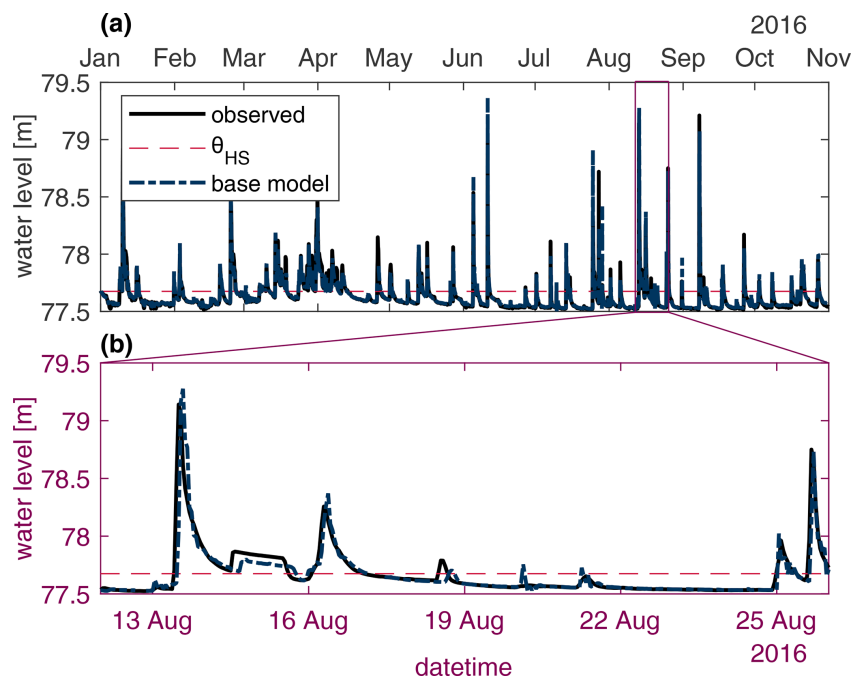

Figure 4. Observed and individual learner stage predictions for the Don River system for all 10 months of an available stage (a) and a $14 \mathrm{~d}$ subset which contains a particularly high stage (b) to better distinguish the two hydrographs. The dashed red line indicates the fixed threshold used to distinguish between typical and high stage values.

demonstrates how the most recent autoregressive input variable is the most important variable for accurate stage predictions (Snieder et al., 2020).

Without accounting for the imbalanced nature of stage data, data-driven models are prone to inadequate performance similar to that of the individual learners described

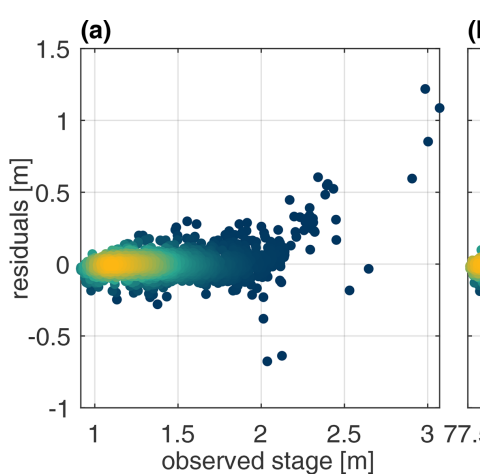

(b)
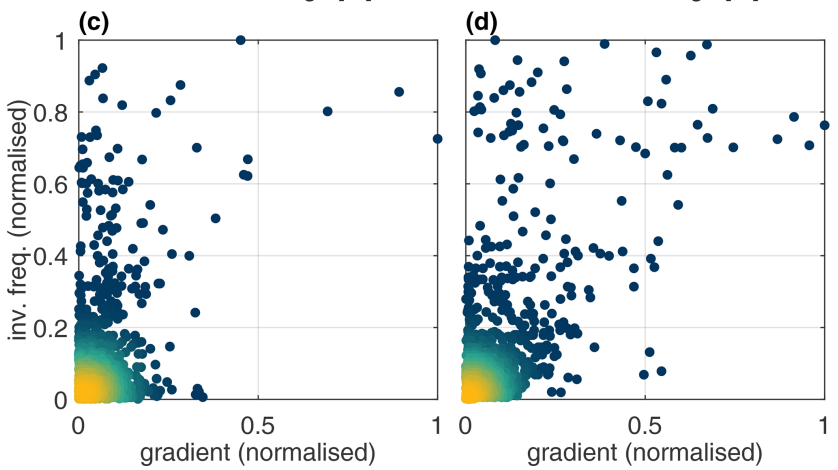

Figure 5. Baseline model residuals versus observed stage for the Bow (a) and Don (b) River systems. Inverse frequency versus gradient across four time steps for the Bow (c) and Don (d) River target variables. Colouring indicates normalised scatter point density.

above. Consequently, such models may not be suitable for flood-related applications such as early flood warning systems. The following section describes and reviews resampling and ensemble methods, which are proposed as solutions to the imbalance problem, which manifests as poor performance on high stage samples, relative to typical stage.

\section{Review and description of methods for handling imbalanced target datasets}

Many strategies have been proposed for handling imbalanced domains, which can be broadly categorised into three approaches: specialised preprocessing, learning methods, and combined methods (Haixiang et al., 2017; Moniz et al., 2018). According to a comprehensive review of imbalanced learning strategies, resampling and ensemble methods are among the most popular techniques employed (Haixiang et al., 2017). Specifically, a review of 527 papers on imbalanced classification found that a resampling technique was used 156 times (Haixiang et al., 2017). From the same review, 218 of the 527 papers used an ensemble technique such as bagging or boosting. Many of the studies reviewed used combinations of available techniques and often propose novel hybrid approaches that incorporate elements from several algorithms. Since it is impractical to compare every 
unique algorithm that has been developed for handling imbalanced data, the scope of this research adheres to relatively basic techniques and combinations of resampling and ensemble methods. The following sections describe the resampling and ensemble methods used in this research. The review attempts to adhere to hydrological studies that feature each of the methods; however, when this is not always possible, examples from other fields are presented.

First, it is important to distinguish between the data imbalance addressed in this study and cost-sensitive imbalance. Imbalance in datasets can be characterised as a combination of two factors: imbalanced distributions of samples across the target domain and imbalanced user interest across the domain. Target domain imbalance is related solely to the native distribution of samples, while cost sensitivity occurs when costs vary across the target domain. While both types of imbalance are relevant to the flow-forecasting application of this research, cost-sensitive methods are complex and typically involve developing a relationship between misprediction and tangible costs, for example, property damage (Toth, 2016). Cost-sensitive learning is outside the scope of this research, which is focused on reducing high stage errors due to the imbalanced nature of the target stage data.

\subsection{Resampling techniques}

Resampling is widely used in machine learning to create subsets of the total available data with which to train models. Resampling is typically used as a data-preprocessing technique (Brown et al., 2005; Moniz et al., 2017a). However, in our research, resampling is embedded in the ensemble algorithms so as to promote diversity amongst the individual learners. This following section discusses examples of resampling, whether used for preprocessing or used within the learning algorithm. Pseudocode for each resampling method is provided in Appendix B.

\subsubsection{Random undersampling}

RUS is performed by subsampling a number of frequent cases equal to the number of infrequent cases, such that there are an even number in each category that achieve a more balanced distribution compared to the original set. As a result, all of the rare cases are used for training, while only a fraction of the normal cases are used. RUS is intuitive for classification problems; for two-class classification, the majority class is undersampled such that the number of samples drawn from each class is equal to the number of samples in the minority class (Yap et al., 2014). However, RUS is less straightforward for regression, as it requires continuous data first to be categorised so as to allow for an even number of samples to be drawn from each category. Categories must be selected appropriately such that they are continuous across the target domain and each category contains a sufficient number of samples to allow for diversity in the resampled dataset (Galar et al., 2013). Undersampling is scarcely used in hydrological forecasting applications, despite seeing widespread use in classification studies. Mahamud et al. (2016) demonstrate an application of fuzzy-based RUS for categorical flood risk support vector machine (SVM)-based classification, which is motivated by the imbalanced nature of flood data. RUS is found to outperform both ROS and the synthetic minority oversampling technique (SMOTE) on average across five locations.

In this research, $N$ available stage samples are categorised into $N_{\mathrm{TS}}$ typical stage and $N_{\mathrm{HS}}$ high stage based on the threshold $\Theta_{\mathrm{HS}}$. The undersampling scheme draws $N_{\mathrm{HS}}$ with replacement from each of the subsets, such that there are an equal number of each category. RUS can be performed with or without replacement; the former provides greater diversity when resampling is repeated several times, and thus this approach is selected for the present research.

\subsubsection{Random oversampling}

ROS simply consists of oversampling rare samples, thus modifying the training sample distribution through duplication (Yap et al., 2014). ROS is procedurally similar to RUS, also aiming to achieve a common number of frequent and infrequent samples. Instead of subsampling the typical stage, high stage values are resampled with replacement so that the number of samples matches that of the typical stage set. The duplication of high stage samples in the training dataset increases their relative contribution to the model's objective function during calibration. Compared to undersampling, oversampling is advantaged such that more samples in the majority class are utilised. The drawbacks of this approach are that there is an increased computational cost. There are few examples of ROS applications in the water resource literature; studies tend to favour SMOTE, which is discussed in the following section. Saffarpour et al. (2015) use oversampling to address the class imbalance of binary flood data; surprisingly, oversampling was found to decrease classification accuracy compared to the raw training dataset. Recently, Zhaowei et al. (2020) applied oversampling for vehicle traffic flow as a response to the imbalance of the training data.

For ROS, as with RUS, $N$ available stage samples are categorised into $N_{\mathrm{TS}}$ typical stage and $N_{\mathrm{HS}}$ high stage samples based on the threshold $\Theta_{\mathrm{HS}}$. The oversampling scheme draws $N_{\text {TS }}$ with replacement from each of the subsets, such that there is an equal number of each category. ROS is distinguished from RUS in that it produces a larger sample set that inevitably contains duplicated high stage values.

\subsubsection{Synthetic minority oversampling technique for regression}

SMOTER is a variation of the SMOTE classification resampling technique introduced by Chawla et al. (2002) that by- 
passes excessive duplication of samples by generating synthetic samples, which, unlike duplication, creates diversity within the ensembles. SMOTE is widely considered an improvement over simple ROS as the increased diversity helps prevent overfitting (Mahamud et al., 2016). For a given sample, SMOTE generates synthetic samples by randomly selecting one of $k$ nearest points, determined using $k$-nearest neighbours (KNN), and sampling a value at a linear distance between the two neighbouring points. The original SMOTE algorithm was developed for classification tasks; Torgo et al. (2013) developed the SMOTER variation, which is an adaptation of SMOTE for regression. SMOTER uses a fixed threshold to distinguish between "rare" and "normal" points. In addition to oversampling synthetic data, SMOTER also randomly undersamples normal values to achieve the desired ratio between rare and normal samples. The use of SMOTE in the development of models that predict river stage has only been attempted recently. Atieh et al. (2017) use two methods for generalisation: dropout and SMOTER; these were applied to ANN models that predicted the flow duration curves for ungauged basins. They found that SMOTER reduced the number of outlier predictions, whereas both approaches resulted in the improved performance of the ANN models. Wu et al. (2020) used SMOTE resampling in combination with AdaBoosted sparse Bayesian models. The combination of these methods resulted in improved model accuracy compared to previous studies using the same dataset. Razali et al. (2020) used SMOTE with various Bayesian network and machine-learning techniques, including decision trees, KNN, and SVM. Each technique is applied to an imbalanced classified flood dataset (flood flow and non-flood flow categories); the SMOTE decision tree model achieved the highest classification accuracy. SMOTE decision trees have also been applied for estimating the pollutant removal efficiency of bioretention cells. Wang et al. (2019a) found that decision trees developed with SMOTE had the highest accuracy for predicting pollutant removal rates; the authors attribute the success of SMOTE to its ability to prevent the majority class from dominating the fitting process. Sufi Karimi et al. (2019) employ SMOTER resampling for stormwater flow prediction models. Their motivation for resampling is flow dataset imbalance and data sparsity. Several configurations are considered with varying degrees of oversampled synthetic and undersampled data. The findings of the study indicate that increasing the oversampling rate tends to improve model performance compared to the nonresampled model, while increasing the undersampling rate produces a marginal improvement. Collectively, these applications of SMOTE affirm its suitability for mitigating the imbalance problem in the flood-forecasting models featured in this research.

SMOTER is adapted in this research following the method described by Torgo et al. (2013). One change in this adaptation is that rare cases are determined using the $\theta_{\mathrm{HS}}$ value, instead of a relevancy function. Similarly, only high values are considered "rare", instead of considering both low and high values to be rare, as in the original algorithm. Oversampling and undersampling are performed at rates of $400 \%$ and $0 \%$, respectively, so as to obtain an equivalent number of normal and rare cases.

\subsection{Ensemble-based techniques}

Ensembles are collections of models (called individual learners), each with variations to the individual learner model type or to the training procedure (Alobaidi et al., 2019). It is well established that ensemble-based methods improve model stability and generalisability (Alobaidi et al., 2019; Brown et al., 2005). Recent advances in ensemble learning have emphasised the importance of diversity in learning (Alobaidi et al., 2019). Diversity can be generated both implicitly and explicitly through a variety of methods, some of which include varying the initial set of model parameters, varying the model topology, varying the training algorithm, and varying the training data (Sharkey, 1996; Brown et al., 2005). The largest source of diversity in the ensembles under study is attributable to varying the training data, which occurs both in the various resampling methods described above and, in some cases, the ensemble algorithms. Only homogeneous ensembles are used in this work; thus, no diversity is obtained by varying the model topology or training algorithm (Zhang et al., 2018; Alobaidi et al., 2019). Ensemble predictions are combined to form a single discrete prediction. Ensembles that are combined to produce discrete predictions have been proven to outperform single models by reducing model bias and variance, thus improving overall model generalisability (Brown et al., 2005; Sharkey, 1996; Shu and Burn, 2004; Alobaidi et al., 2019). This has contributed to their widespread application in hydrological modelling (Abrahart et al., 2012). In some cases, ensembles are not combined, and the collections of predictions are used to estimate the uncertainty associated with the diversity between ensemble members (Tiwari and Chatterjee, 2010; Abrahart et al., 2012). While this approach has obvious advantages, it is not possible for all types of ensembles, such as the boosting methods, which are also used in this research. Thus, this research combines ensembles to aid comparison across the different resampling and ensemble methods used.

There are many distinct methods for creating ensemble methods. The purpose of this paper is not to review all ensemble algorithms, but rather to compare three ensemble methods that commonly appear in the literature: bagging, adaptive boosting, and gradient boosting. A fourth method, randomised weights and biases, which does not qualify as an ensemble technique due to the absence of repeated resampling, is also included in the ensemble comparison because of its widespread use. While several studies have provided comparisons of ensemble methods, none of these studies has explicitly studied their effects on high stage prediction or their 
combination with resampling strategies, which is common in applications outside of flow forecasting.

Methods that aim to improve generalisability have shown promise in achieving improved prediction on high stage, which may be scarcely represented in training data. However, to the knowledge of the authors, no research has explicitly evaluated the efficacy of ensemble-based methods for improving high stage accuracy. Applications of ensemble methods for improving performance of imbalanced target variables have been thoroughly studied in the classification literature. Several classification studies have demonstrated how ensemble techniques can improve prediction accuracy for imbalanced classes (Galar et al., 2012; López et al., 2013; Díez-Pastor et al., 2015b, a; Błaszczyński and Stefanowski, 2015). Such methods are increasingly being adapted for regression problems, which is typically achieved by projecting continuous data into a classification dataset (Moniz et al., 2017b, a; Solomatine and Shrestha, 2004). Pseudocode for each of the ensemble algorithms used in this research is provided in Appendix B.

\subsubsection{Randomised weights and biases}

While not technically a form of ensemble learning, repeatedly randomising the weights and biases of ANNs is one of the simplest and most common methods for achieving diversity among a collection of models; thus, it acts as a good comparison point for the proceeding ensemble methods (Brown et al., 2005). In this method, members are only distinguished by the randomisation of the initial parameter values (i.e. the initial weights and biases for ANNs in this research) used for training. For this method, an ensemble of ANNs is trained, each member having a different randomised set of initial weights and biases. Thus, when trained, each ensemble member may converge to different final weight and bias values. Ensemble members are combined through averaging. This technique is often used, largely to alleviate variability in training outcomes and uncertainty associated with the initial weight and bias parameterisation (Shu and Burn, 2004; de Vos and Rientjes, 2005; Fleming et al., 2015; Barzegar et al., 2019). Despite its simplicity, this method has been demonstrated to produce considerable improvements in performance when compared to a single ANN model, even outperforming more complex ensemble methods (Shu and Burn, 2004). The weights and biases of each ANN are initialised using the default initialisation function in MATLAB, and an ensemble size of 20 is used.

\subsubsection{Bagging}

Bagging is a widely used ensemble method first introduced in Breiman (1996). Bagging employs the bootstrap resampling method, which consists of sampling with replacement, to generate subsets of data on which to train ensemble members. The ensemble members are combined through simple averaging to form discrete predictions. Bagging is a proven ensemble method in flood prediction studies and has been widely applied and refined for both spatial and temporal prediction since its introduction by Breiman (1996). Chapi et al. (2017) use bagging with logistic model trees (LMTs) as the individual learners to predict spatial flood susceptibility. The bagging ensemble is found to outperform standalone LMTs in addition to logistic regression and Bayesian logistic regression. For a similar flood susceptibility prediction application, Chen et al. (2019) use Bagging with Reduced Error Pruning Trees (REPTree) as the base learners. The bagged models are compared to random subspace ensembles; both ensemble methods perform better than the standalone REPTree models, with the random subspace model slightly outperforming the bagged ensemble. Anctil and Lauzon (2004) compared five generalisation techniques in the development of ANNs for flow forecasting. They combined bagging, boosting, and stacking with stop training and Bayesian regularisation, making a total of nine model configurations. They found that stacking, bagging, and boosting all resulted in improved model performance, ultimately recommending the use of the last two in conjunction with either stop training or Bayesian regularisation. Ouarda and Shu (2009) compared stacking and bagging ANN models against parametric regression for estimating low-flow quantiles for the summer and winter seasons and found higher performance in ANN models (single and ensemble) compared to traditional regression models (Ouarda and Shu, 2009). Cannon and Whitfield (2002) applied bagging to MLP-ANN models for predicting flow and found that bagging helped create the bestperforming ensemble ANN. Shu and Burn (2004) evaluated six approaches for creating ANN ensembles for regional flood frequency flood analysis, including bagging combined with either simple averaging or stacking; bagging resulted in higher performance compared to the basic ensemble method. In a later study, Shu and Ouarda (2007) used bagging and simple averaging to create ANN ensembles for estimating regional flood quantiles at ungauged sites. Implementing bagging is uncomplicated: a description of the algorithm is described in its original appearance (Breiman, 1996). This research uses a bagging ensemble of 20 members.

\subsubsection{Adaptive boosting for regression}

The AdaBoost algorithm was originally developed by Freund and Schapire (1996) for classification problems. The algorithm has undergone widespread adaptation and its popularity has led to the development of many variations which typically introduce improvements in performance and efficiency and are expanded for regression problems. This study uses the AdaBoost.RT variation (Solomatine and Shrestha, 2004; Shrestha and Solomatine, 2006). Broadly put, the AdaBoost algorithm begins by training an initial model. The following model in the ensemble is trained using a resampled or reweighted training set, based on the residual error of the 
previous model. This process is typically repeated until the desired ensemble size is achieved or a stopping criterion is met. Predictions are obtained by a weighted combination of the ensemble members, where model weights are a function of their overall error.

Similarly to bagging, there are many examples of AdaBoost applications for hydrological prediction. Solomatine and Shrestha (2004) compared various forms of AdaBoost against bagging in models predicting river flows and found AdaBoost.RT to outperform bagging. In a later study, the same authors compared the performance of AdaBoosted M5 tree models against ANN models for various applications, including predicting river flows in a catchment; they found higher performance in models that used the AdaBoost.RT algorithm compared to single ANNs (Shrestha and Solomatine, 2006). Liu et al. (2014) used AdaBoost.RT for calibrating process-based rainfall-runoff models and found improved performance over the single model predictions. Wu et al. (2020) compared boosted ensembles against bagged ensembles for predicting hourly streamflow and found that the combination of AdaBoost (using resampling) and Bayesian model averaging gave the highest performance.

The variant of AdaBoost in this research follows the algorithm AdaBoost.RT proposed by Solomatine and Shrestha (2004) and Shrestha and Solomatine (2006). This algorithm has three hyperparameters. The relative error threshold parameter is selected as the 80th percentile of the residuals of the individual learner, and 20 ensemble members are trained. AdaBoost can be performed using either resampling or reweighting (Shrestha and Solomatine, 2006); resampling is used in this research as it has been found to typically outperform reweighting (Seiffert et al., 2008). Recently, several studies have independently proposed a modification to the original AdaBoost.RT algorithm by adaptively calculating the relative error threshold value for each new ensemble member (Wang et al., 2019b; Li et al., 2020). This modification to the algorithm was generally found to be detrimental to the performance of the models in the present research; thus, the static error threshold described in the original algorithm description was used (Solomatine and Shrestha, 2004).

\subsubsection{Least-squares boosting}

LSBoost is a variant of gradient boosting, which is an algorithm that involves training an initial model, followed by a sequence of models that are each trained to predict the residuals of the previous model in the sequence. This is in contrast to the AdaBoost method, which uses the model residuals to inform a weighted sampling scheme for subsequent models. The prediction at a given training iteration is calculated by the weighted summation of the already trained model(s) from the previous iterations. For LSBoost weighting is determined by a least-squares loss function; other variants of gradient boosting use a different loss function (Friedman, 2000).
Gradient-boosting algorithms have previously been used to improve efficiency and accuracy for hydrological forecasting applications. Ni et al. (2020) use the gradient-boosting variant XGBoost, which uses decision trees (DTs) as the individual learners in combination with a Gaussian mixture model (GMM) for streamflow forecasting. The GMM is used to cluster streamflow data, and an XGBoost ensemble is fitted to each cluster. Clustering streamflow data into distinct subsets for training is sometimes used as an alternative to resampling; their purpose is similar to that of resampling, which is to change the training sample distribution (Wang et al., 2006). The combination of XGBoost and GMM is found to outperform standalone SVM models. Erdal and Karakurt (2013) developed gradient-boosted regression trees and ANNs for predicting daily streamflow and found gradient-boosted ANNs to have higher performance than the regression tree counterparts. Worland et al. (2018) use gradient-boosted regression trees to predict annual minimum $7 \mathrm{~d}$ streamflow at 224 unregulated sites; performance is found to be competitive with several other types of data-driven models. Zhang et al. (2019) use the Online XGBoost gradient-boosting algorithm for regression tree models to simulate streamflow and found that it outperformed many other data-driven and lumped hydrological models. Papacharalampous et al. (2019) use gradient boosting with regression trees and linear models, which are compared against several other model types for physically based hydrological model quantile regression post-processing. Neither of the gradient-boosting models outperforms the other regression models, and a uniformly weighted ensemble of all other model types typically outperforms any individual model type. These examples of gradient boosting affirm its capability for improving performance compared to the single model comparison as well as other machine-learning models. However, none of these studies uses gradient boosting with ANNs as the individual learner. Moreover, these studies do not examine the effects of gradient boosting on model behaviour within the context of the imbalance problem. Therefore, we use LSBoost to study its efficacy for improving high stage performance.

The implementation of LSBoost in this research is unchanged from the original algorithm (Friedman, 2000). The algorithm has two hyperparameters: the learning rate which scales the contribution of each new model and the number of boosts. A learning rate of 1 is used, and the number of an ensemble size of 20 is used.

\subsection{Hybrid methods}

The resampling and training strategies reviewed above can be combined to further improve model performance on imbalanced data; numerous algorithms have been proposed in the literature that embed resampling schemes in ensemble learning methods. Galar et al. (2012) describe a taxonomy and present a comprehensive comparison of such algorithms 
for classification problems. Many of these algorithms effectively present minor improvements or refinements to popular approaches. Alternatively to implementing every single unique algorithm for training ensembles, the present research suggests employing a systematic approach to combine preprocessing resampling and ensemble training algorithms in a modular fashion; such combinations are referred to as "hybrid methods". Hybrid methods hope to achieve the benefits of both standalone methods: improved performance on a high stage while maintaining good generalisability. Thus, in this research, every permutation of resampling (RUS, ROS, and SMOTER) and ensemble methods (RWB, bagging, AdaBoost, and LSBoost) is evaluated, resulting in 12 unique hybrid methods. For resampling combinations with RWB ensembles, the resampling is performed once; thus, diversity is only obtained from the initialisation of the ANN. This combination is equivalent to evaluating each resampling technique individually to provide a basis for comparison with resampling repeated for each ensemble member, as used in the other ensemble-based configurations. For combinations of resampling with bagging, AdaBoost, and LSBoost, the resampling procedure is performed for training each new ensemble member. One non-intuitive hybrid case is the combination of SMOTER with AdaBoost, because the synthetically generated samples do not have predetermined error weights. A previous study has recommended assigning the initial weight value to synthetic samples (Díez-Pastor et al., 2015a). However, this research proposes that synthetic sample weights are calculated in the same manner as the synthetic samples (e.g. based on the randomly interpolated point between a sample and a random neighbouring point). Thus, if two samples with relatively high weights are used to generate a synthetic sample, the new sample will have a similar weight.

The hyperparameters for each of the resampling and ensemble methods employed in this study are listed in Table 4. Every ensemble uses the ANN described in Sect. 2.2 as the individual learner. The hyperparameters of the individual learner are kept the same throughout all of the ensemble methods to allow for a fair comparison (Shu and Burn, 2004) (excluding of course the number of epochs, which is determined through validation stop training).

\subsection{Model implementation and evaluation}

All aspects of this work are implemented in MATLAB 2020a. The Neural Network Toolbox was used to train the baseline ANN models. The resampling and ensemble algorithms used in this research were programmed by the authors and are available upon request; the pseudocode for each method is available in Appendix B.

\subsubsection{Performance assessment}

The challenges of training models on imbalanced datasets outlined in Sect. 1 and evaluating model performance are one and the same: many traditional performance metrics (e.g. MSE, CE) are biased towards the most frequent stage values, and the metrics are insensitive to changes in high stage accuracy. In fact, despite their widespread use, these metrics are criticised in the literature. For example, ANN models for sunspot prediction produced a lower RMSE (equivalent to a $\mathrm{CE}$ when used on datasets with the same observed mean) compared to conventional models but were found to have no predictive value (Abrahart et al., 2007). Similarly, CE values may be misleadingly favourable if there is significant observed seasonality (Ehret and Zehe, 2011). CE is also associated with the underestimation of peak flows, volume balance errors, and undersized variability (Gupta et al., 2009; Ehret and Zehe, 2011). Zhan et al. (2019) suggest that CE is sensitive to peak flows due to the square term. This assertion is correct while comparing two samples; however, when datasets are imbalanced, the errors of a typical stage overwhelm those of a high stage. Ehret and Zehe (2011) evaluate the relationship between phase error and RMSE using triangular hydrographs; their study shows how RMSE is highly sensitive to minor phase errors; however, when a hydrograph has a phase and amplitude error, RMSE is much more sensitive to overpredictions compared to underpredictions.

The CE, commonly known as the Nash-Sutcliffe efficiency, is given by the following formula:

$\mathrm{CE}=1-\frac{\sum(q(t)-\hat{q}(t))^{2}}{\sum(q(t)-\bar{q})^{2}}$

where $q$ is the observed stage, $\hat{q}$ is the predicted stage, and $\bar{q}$ is the mean observed stage.

The persistence index (PI) is a measure similar to CE, but instead of normalising the sum of squared error of a model based on the observed variance, it is normalised based on the sum of squared error between the target variable and itself, lagged by the lead time of the forecast model (referred to as the naive model). Thus, the CE and PI range from an optimum value of 1 to $-\infty$, with values of 0 corresponding to models that are indistinguishable from the observed mean and naive models, respectively. Since both models use antecedent input variables with lag times equal to the forecast length, PI is a useful indicator of over-reliance on this input variable, which has been associated with peak stage timing error (de Vos and Rientjes, 2009). Furthermore, the PI measure overcomes some of the weaknesses of CEs, such as a misleadingly high value for seasonal watersheds. Moreover, PI is effective in identifying when models become overreliant on autoregressive inputs, as the model predictions will resemble those of the naive model. PI is given by the follow- 
Table 4. Summary of ensemble methods and hyperparameters.

\begin{tabular}{|c|c|c|c|}
\hline Type & Complete name & Short form & Hyperparameters \\
\hline \multirow[t]{6}{*}{ Resampling } & Random undersampling & RUS & Rare case threshold $\left(\theta_{\mathrm{HS}}\right)=80$ th percentile stage \\
\hline & Random oversampling & ROS & Rare case threshold $\left(\theta_{\mathrm{HS}}\right)=80$ th percentile stage \\
\hline & $\begin{array}{l}\text { Synthetic minority oversampling tech- } \\
\text { nique }\end{array}$ & SMOTER & Rare case threshold $\left(\theta_{\mathrm{HS}}\right)=80$ th percentile stage \\
\hline & & & Oversampling percentage $=400 \%$ \\
\hline & & & Undersampling percentage $=0 \%$ \\
\hline & & & $K$-nearest neighbours $=10$ \\
\hline \multirow[t]{4}{*}{ Ensemble } & Randomised initial weights and biases & RWB & - \\
\hline & Bootstrap aggregating & Bagging & Combination weighting: uniform \\
\hline & $\begin{array}{l}\text { Adaptive boosting (for regression using } \\
\text { error thresholding) }\end{array}$ & AdaBoost & $\begin{array}{l}\text { Error threshold }=80 \text { th percentile of base model errol } \\
\text { Resampling/reweighting = resampling }\end{array}$ \\
\hline & Least-squares boosting & LSBoost & $\begin{array}{l}\text { Learning rate }=1 \\
\text { Combination weight }=\text { least squares }\end{array}$ \\
\hline
\end{tabular}

ing formula:

$\mathrm{PI}=1-\frac{\sum(q(t)-\hat{q}(t))^{2}}{\sum(q(t)-q(t-L))^{2}}$,

where $L$ is the lead time of the forecast.

In order to quantify changes in model performance on high stage, both the CE and PI measures are calculated for typical stage (TS) and high stage (HS) (Crochemore et al., 2015). The resampling methods are expected to improve the high stage CE at the expense of CE for typical stage, while ensemble methods are expected to produce an outright improvement in model generalisation, reflected by reduced loss in performance between the calibration and test data partitions. Thus, the objective of this research is to find model configurations with improved performance on high stage while maintaining strong performance overall. TS and HS performance metrics are calculated based only on the respective observed stage. For example, the $\mathrm{CE}$ for high stage is calculated by

$\mathrm{CE}_{\mathrm{HS}}=1-\frac{\sum\left(q_{\mathrm{HS}}(t)-\hat{q}_{\mathrm{HS}}(t)\right)^{2}}{\sum\left(q_{\mathrm{HS}}(t)-\bar{q}_{\mathrm{HS}}\right)^{2}}$,

where $q_{\mathrm{HS}}$ is given by

$q_{\mathrm{HS}}=q \mid q \geq \theta_{\mathrm{HS}}$.

The performances for $\mathrm{CE}_{\mathrm{TS}}, \mathrm{PI}_{\mathrm{HS}}$, and $\mathrm{PI}_{\mathrm{TS}}$ are calculated in the same manner, substituting $q_{\mathrm{TS}}(t)$ for $q_{\mathrm{HS}}(t)$ in Eq. (4) for HS calculations and using Eq. (2) in place of Eq. (1) for PI calculations.

\subsection{2 $K$-fold cross-validation}

The entire available dataset is used for both training and testing by the use of KFCV, a widely used cross-validation method (Hastie et al., 2009; Bennett et al., 2013; Solomatine and Ostfeld, 2008; Snieder et al., 2020). Ten folds are used in total: eight folds for calibration and two for testing. Of the eight calibration folds, six are used for training, while two are used for early stopping. When performance is reported as a single value, it refers to the mean model performance of the respective partition across $K$-folds. It is important to distinguish between the application of KFCV for evaluation (as used in this research) as opposed to using KFCV for producing ensembles, in which an ensemble of models is trained based on a KFCV data-partitioning scheme (Duncan, 2014).

\section{Results}

This section provides a comparison of the performance of each of the methods described throughout Sect. 3 applied to the Bow and Don River watersheds, which are described in Sect. 2.1. Changes to model performance are typically discussed relative to the individual learner (see Sect. 2.2) unless explicit comparisons are specified. First, the results of a grid-search analysis of ensemble size are provided. Next, a general overview and comparison of the results are presented, followed by detailed comparison of the resampling and ensemble methods. Finally, the effects that varying the HS threshold and ensemble size have on resampling and high stage performance are evaluated for the bagging and SMOTER-Bagging models.

Figure 6 illustrates the change in test performance as the ensemble size increases from 2 to 100 for each river. This grid search is performed only for the base ensemble methods (RWB, bagging, AdaBoost, and LSBoost) without any resampling. The Bow River results indicate that AdaBoost and LSBoost tend to favour a small ensemble size (2-15 members), whereas the generalisation of RWB and bagging im- 


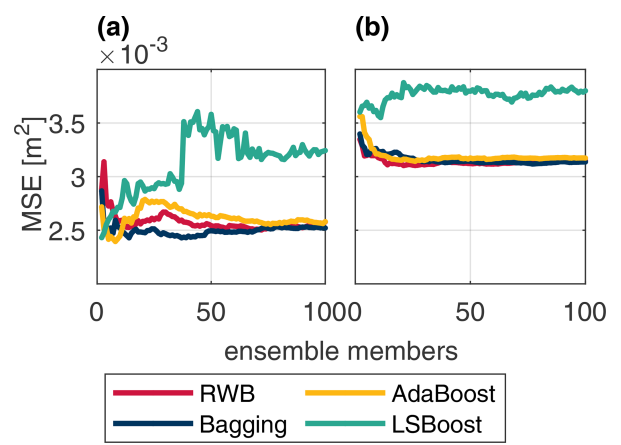

Figure 6. Test MSE across ensemble size for RWB (red), bagging (blue), AdaBoost (yellow), and LSBoost (green) for the Don River (a) and Bow River (b).

proves with a larger size ( $>20$ members). The performance of LSBoost rapidly deteriorates as the ensemble size grows, likely as the effects of overfitting become more pronounced. Similar results are obtained for the Don, except that RWB, bagging, and AdaBoost all improve with larger ensemble size, while LSBoost performs worse than all other ensembles, even for small ensemble sizes. Similar to the Bow, a larger ensemble size ( $>20$ members) produces favourable MSE.

Figures 7 and 8 show the CE and PI box-whisker plots for the Bow and Don rivers, respectively. These figures show the performance of the test dataset, across the $K$-folds, for each resampling, ensemble, and hybrid technique as well as the individual learner. The performance metrics are calculated for the entire dataset, the HS values, and the TS values. Models with a larger range have more variable performance when evaluated across different subsets of the available data.

The average performances for each resampling, ensemble, and hybrid method for the Bow and Don River models are shown in Tables 5 and 6, respectively, which list the CE and PI for the entire dataset as well as the TS and HS datasets. The ensemble results for each KFCV fold were combined using a simple arithmetic average. The results have been separated into different categories: each section starts with the ensemble technique (either RWB, bagging, AdaBoost, or LSBoost), followed by the three hybrid variations (RUS-, ROS-, or SMOTER-). The calibration (training and validation) performance is indicated in parentheses and italics, followed by the test performance. Comparing both the calibration and test performance is useful since it provides a sense of overfitting and hence generalisation. For example, an improvement in calibration performance and decrease in test performance suggest that the model has been overfitted. In contrast, improvements to both partitions indicate favourable model generalisation. The best performing model (based on testing performance) has been highlighted in bold text for each performance metric, CE and PI, for both watersheds.

Based on the CE values in Figs. 7-8 and Tables 5-6, the majority of the Bow and Don River models achieve "accept-
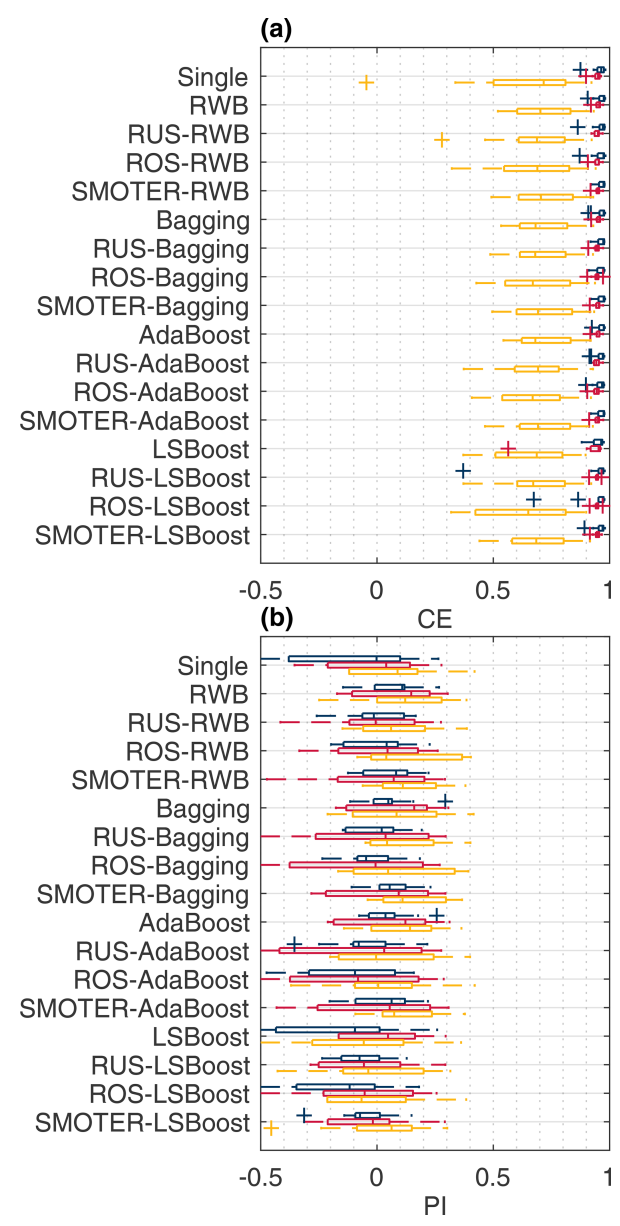

Figure 7. Overall (blue), typical stage (red), and high stage (yellow) CE (a) and PI (b) for the Bow River models.

able" prediction accuracy (as defined by Mosavi et al., 2018). Values of $\mathrm{CE}_{\mathrm{TS}}$ and $C E_{\mathrm{HS}}$ are both lower than the $\mathrm{CE}$, which is to be expected as the stage variance of each subset is lower than that of the set of all stage values. For the Bow River models, the $\mathrm{CE}$ and $\mathrm{CE}_{\mathrm{TS}}$ values are consistently higher than the $\mathrm{CE}_{\mathrm{HS}}$; this is attributable to the high seasonality of the watershed producing a misleadingly high value for $\mathrm{CE}$ due to the high variance of stage throughout the year, as discussed in Sect. 3.4.1. The $\mathrm{CE}_{\mathrm{HS}}$ values also have higher variability compared to the overall $\mathrm{CE}$ and $\mathrm{CE}_{\mathrm{TS}}$, as shown in Fig. 7a. In contrast, for the Don River models, the difference in CE, $\mathrm{CE}_{\mathrm{TS}}$, and $\mathrm{CE}_{\mathrm{HS}}$ is less pronounced, whereas the $\mathrm{CE}$ (for the entire dataset) is typically higher, as expected, than both the $\mathrm{CE}_{\mathrm{TS}}$ and $\mathrm{CE}_{\mathrm{HS}}$; the difference between $\mathrm{CE}_{\mathrm{TS}}$ and $\mathrm{CE}_{\mathrm{HS}}$ is low, as demonstrated in the mean and range of the boxwhisker plots in Fig. 8a. Unlike the Bow River, the Don River does not exhibit notable seasonality, resulting in a smaller difference between the HS and TS.

Values of PI are typically lower than for CE for both watersheds. The Bow River models obtain PI values centred around 0 (see Fig. 7b), indicating that only some of the model 
Table 5. Mean CE and PI scores for all, typical, and high stage for the Bow River ensembles; the highest scores are shown in bold, and the calibration scores are italicised and enclosed by parentheses.

\begin{tabular}{lrrrrrrr}
\hline Label & $\mathrm{CE}$ & $\mathrm{CE}_{\mathrm{TS}}$ & $\mathrm{CE}_{\mathrm{HS}}$ & $\mathrm{PI}$ & $\mathrm{PI}_{\mathrm{TS}}$ & $\mathrm{PI}_{\mathrm{HS}}$ \\
\hline Base model & $(0.967) 0.954$ & $(0.954) 0.944$ & $(0.829) 0.617$ & $(0.182)-0.166$ & $(0.111)-0.0593$ & $(0.227)-0.175$ \\
\hline RWB & $(0.974) 0.962$ & $(0.96) 0.951$ & $(0.865) 0.718$ & $(0.331) \mathbf{0 . 0 7 3 1}$ & $(0.229) 0.0856$ & $(0.392) 0.128$ \\
RUS-RWB & $(0.972) 0.956$ & $(0.954) 0.947$ & $(0.863) 0.68$ & $(0.286)-0.0505$ & $(0.116)-0.013$ & $(0.384) 0.015$ \\
ROS-RWB & $(0.973) 0.957$ & $(0.955) 0.947$ & $(0.87) 0.681$ & $(0.312)-0.0266$ & $(0.125) 0.00468$ & $(0.418) 0.0454$ \\
SMOTER-RWB & $(0.974) \mathbf{0 . 9 6 3}$ & $(0.957) 0.948$ & $(0.871) \mathbf{0 . 7 2}$ & $(0.329) 0.0524$ & $(0.176) 0.0168$ & $(0.417) 0.139$ \\
\hline Bagging & $(0.973) 0.961$ & $(0.96) \mathbf{0 . 9 5 2}$ & $(0.86) 0.709$ & $(0.32) 0.0503$ & $(0.234) \mathbf{0 . 0 8 8 6}$ & $(0.372) 0.0887$ \\
RUS-Bagging & $(0.972) 0.961$ & $(0.955) 0.945$ & $(0.867) 0.715$ & $(0.298) 0.00346$ & $(0.119)-0.0403$ & $(0.399) 0.116$ \\
ROS-Bagging & $(0.973) 0.959$ & $(0.954) 0.943$ & $(0.873) 0.696$ & $(0.312)-0.0374$ & $(0.111)-0.0851$ & $(0.425) 0.0896$ \\
SMOTER-Bagging & $(0.974) 0.962$ & $(0.957) 0.948$ & $(0.873) 0.719$ & $(0.333) 0.0511$ & $(0.17) 0.018$ & $(0.427) \mathbf{0 . 1 4 4}$ \\
\hline AdaBoost & $(0.974) 0.963$ & $(0.96) 0.95$ & $(0.865) 0.719$ & $(0.327) 0.0465$ & $(0.22) 0.0488$ & $(0.389) 0.112$ \\
RUS-AdaBoost & $(0.972) 0.959$ & $(0.954) 0.942$ & $(0.865) 0.693$ & $(0.288)-0.0642$ & $(0.107)-0.105$ & $(0.39) 0.0509$ \\
ROS-AdaBoost & $(0.972) 0.956$ & $(0.951) 0.942$ & $(0.872) 0.673$ & $(0.291)-0.114$ & $(0.052)-0.109$ & $(0.424)-0.0307$ \\
SMOTER-AdaBoost & $(0.974) 0.962$ & $(0.957) 0.947$ & $(0.872) 0.714$ & $(0.331) 0.0259$ & $(0.166)-0.00642$ & $(0.425) 0.121$ \\
\hline LSBoost & $(0.974) 0.948$ & $(0.958) 0.907$ & $(0.869) 0.666$ & $(0.328)-0.504$ & $(0.189)-0.786$ & $(0.403)-0.104$ \\
RUS-LSBoost & $(0.97) 0.904$ & $(0.952) 0.944$ & $(0.854) 0.364$ & $(0.246)-0.718$ & $(0.0643)-0.0609$ & $(0.35)-0.824$ \\
ROS-LSBoost & $(0.973) 0.929$ & $(0.952) 0.944$ & $(0.875) 0.517$ & $(0.304)-0.425$ & $(0.0638)-0.0757$ & $(0.435)-0.431$ \\
SMOTER-LSBoost & $(0.973) 0.958$ & $(0.954) 0.946$ & $(0.868) 0.684$ & $(0.3)-0.0522$ & $(0.117)-0.0255$ & $(0.401) 0.00239$ \\
\hline
\end{tabular}

Table 6. Mean CE and PI scores for all, typical, and high stage for the Don River ensembles; the highest scores are shown in bold, and the calibration scores are italicised and enclosed by parentheses.

\begin{tabular}{lrrrrrrr}
\hline Label & $\mathrm{CE}$ & $\mathrm{CE}$ & $\mathrm{CE}_{\mathrm{TS}}$ & $\mathrm{PI}$ & $\mathrm{PI}_{\mathrm{TS}}$ & $\mathrm{PI}_{\mathrm{HS}}$ \\
\hline Base model & $(0.86) 0.781$ & $(0.782) 0.664$ & $(0.677) 0.511$ & $(0.716) 0.592$ & $(0.0197)-0.213$ & $(0.74) 0.61$ \\
\hline RWB & $(0.873) 0.806$ & $(0.814) 0.755$ & $(0.705) 0.572$ & $(0.744) 0.641$ & $(0.165) \mathbf{0 . 0 9 4 4}$ & $(0.763) 0.654$ \\
RUS-RWB & $(0.853) 0.792$ & $(0.638) 0.588$ & $(0.685) 0.555$ & $(0.704) 0.615$ & $(-0.585)-0.63$ & $(0.746) 0.645$ \\
ROS-RWB & $(0.864) 0.799$ & $(0.629) 0.488$ & $(0.715) 0.584$ & $(0.726) 0.624$ & $(-0.632)-0.991$ & $(0.771) 0.665$ \\
SMOTER-RWB & $(0.866) 0.795$ & $(0.642) 0.552$ & $(0.715) 0.57$ & $(0.729) 0.618$ & $(-0.573)-0.749$ & $(0.771) 0.656$ \\
\hline Bagging & $(0.869) \mathbf{0 . 8 0 8}$ & $(0.811) \mathbf{0 . 7 5 7}$ & $(0.696) 0.581$ & $(0.736) \mathbf{0 . 6 5}$ & $(0.154) 0.0875$ & $(0.755) 0.663$ \\
RUS-Bagging & $(0.864) 0.805$ & $(0.676) 0.609$ & $(0.706) \mathbf{0 . 5 8 5}$ & $(0.726) 0.638$ & $(-0.433)-0.502$ & $(0.764) \mathbf{0 . 6 6 8}$ \\
ROS-Bagging & $(0.858) 0.795$ & $(0.553) 0.271$ & $(0.716) 0.584$ & $(0.712) 0.618$ & $(-1.14)-1.41$ & $(0.771) 0.665$ \\
SMOTER-Bagging & $(0.865) 0.798$ & $(0.604) 0.526$ & $(0.718) 0.581$ & $(0.729) 0.623$ & $(-0.705)-0.888$ & $(0.774) 0.662$ \\
\hline AdaBoost & $(0.87) 0.803$ & $(0.807) 0.744$ & $(0.698) 0.567$ & $(0.737) 0.637$ & $(0.136) 0.0393$ & $(0.758) 0.651$ \\
RUS-AdaBoost & $(0.857) 0.787$ & $(0.658) 0.53$ & $(0.694) 0.553$ & $(0.712) 0.613$ & $(-0.51)-0.888$ & $(0.754) 0.646$ \\
ROS-AdaBoost & $(0.864) 0.793$ & $(0.604) 0.516$ & $(0.718) 0.575$ & $(0.726) 0.616$ & $(-0.725)-1.07$ & $(0.773) 0.658$ \\
SMOTER-AdaBoost & $(0.867) 0.801$ & $(0.667) 0.578$ & $(0.715) 0.584$ & $(0.732) 0.629$ & $(-0.46)-0.743$ & $(0.771) 0.665$ \\
\hline LSBoost & $(0.869) 0.746$ & $(0.813) 0.741$ & $(0.696) 0.446$ & $(0.736) 0.555$ & $(0.169) 0.0719$ & $(0.755) 0.567$ \\
RUS-LSBoost & $(0.835) 0.715$ & $(0.744) 0.685$ & $(0.625) 0.419$ & $(0.67) 0.513$ & $(-0.128)-0.207$ & $(0.697) 0.548$ \\
ROS-LSBoost & $(0.871) 0.759$ & $(0.761) 0.716$ & $(0.708) 0.472$ & $(0.738) 0.561$ & $(-0.0738)-0.0931$ & $(0.766) 0.579$ \\
SMOTER-LSBoost & $(0.871) 0.787$ & $(0.775) 0.695$ & $(0.707) 0.537$ & $(0.74) 0.599$ & $(0.00723)-0.0914$ & $(0.765) 0.62$ \\
\hline
\end{tabular}

configurations perform with greater accuracy than the naive model, meaning that a timing error exists. The box-whisker plots of each ensemble method do not show a clear trend (with respect to the mean value or range) when comparing the $\mathrm{PI}, \mathrm{PI}_{\mathrm{TS}}$, and $\mathrm{PI}_{\mathrm{HS}}$ : the mean and range are similar for all variants tested.
The Don River models have positive PI values of approximately 0.6 , indicating a lower reliance on autoregressive input variables when compared to the Bow River. In contrast to the Bow River, there is a notable difference between the PI metrics: the $\mathrm{PI}_{\mathrm{TS}}$ has a lower mean value and higher variance (see Fig. 8b) than the PI (for the entire dataset) and the $\mathrm{PI}_{\mathrm{HS}}$. These lower $\mathrm{PI}_{\mathrm{TS}}$ are due to the low variability (steadiness) 

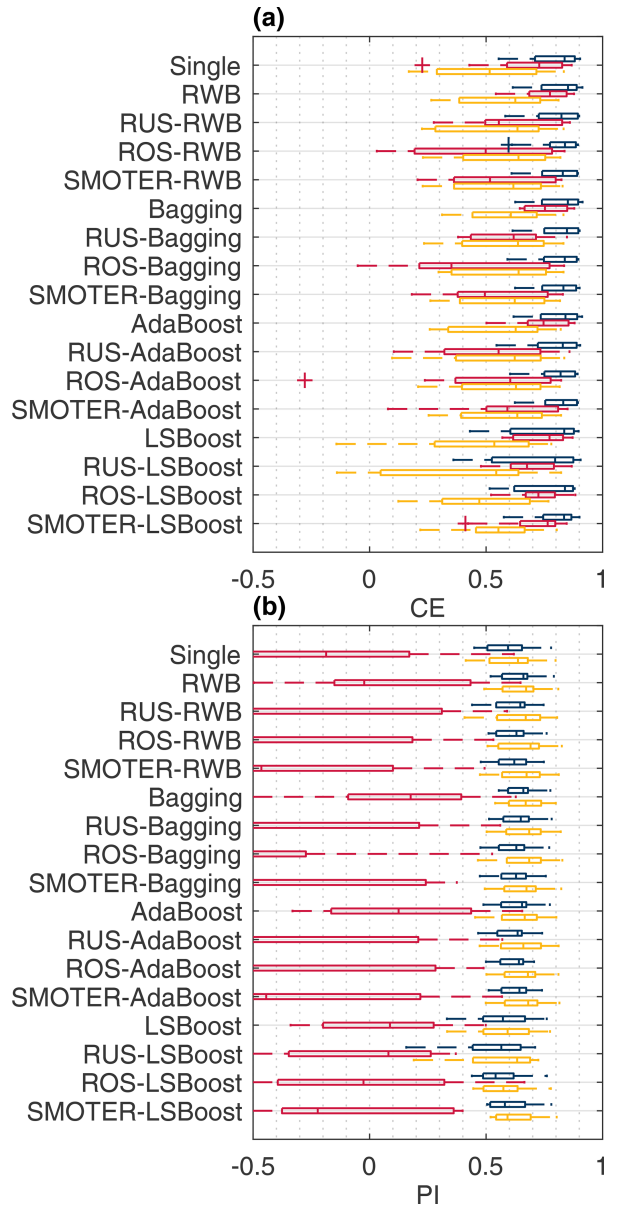

Figure 8. Overall (blue), typical stage (red), and high stage (yellow) CE (a) and PI (b) for the Don River models. (a) $\mathrm{CE}$

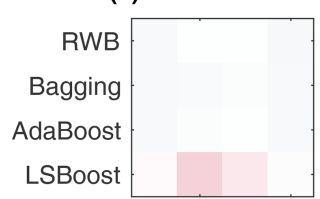

(d) PI

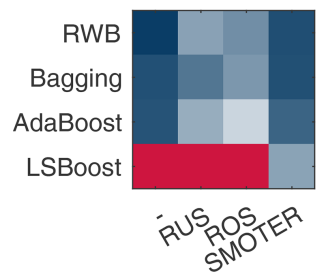

(b) $\mathrm{CE}_{\mathrm{TS}}$

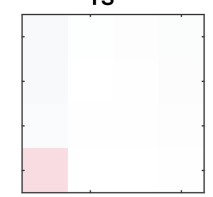

(e) $\mathrm{PI}_{\mathrm{TS}}$

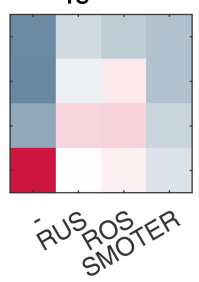

(c) $\mathrm{CE}_{\mathrm{HS}}$

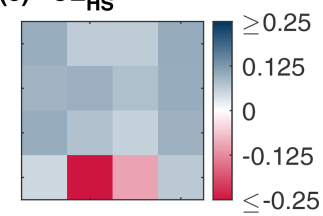

(f) $\mathrm{PI}_{\mathrm{HS}}$

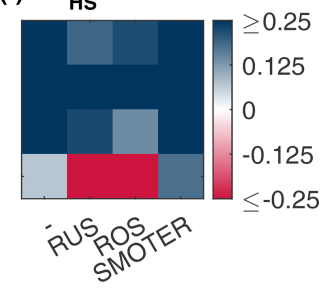

Figure 9. Change in (absolute) performance of $\mathrm{CE}$ (a), $\mathrm{CE}_{\mathrm{TS}}$ (b), $\mathrm{CE}_{\mathrm{HS}}$ (c), $\mathrm{PI}(\mathbf{d}), \mathrm{PI}_{\mathrm{TS}}(\mathbf{e})$, and $\mathrm{PI}_{\mathrm{HS}}$ (f) produced by combinations of resampling (listed along the $x$ axis) and ensemble (listed along the $y$ axis) methods for the Bow River models. (a) CE

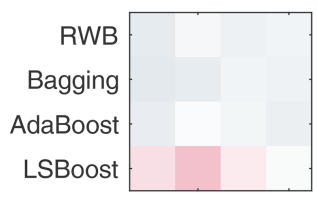

(d) $\mathrm{PI}$

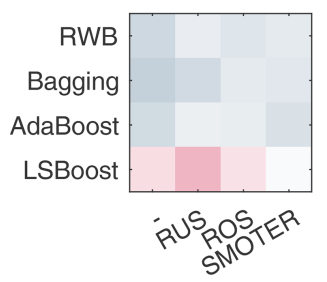

(b) $\mathrm{CE}_{\mathrm{TS}}$

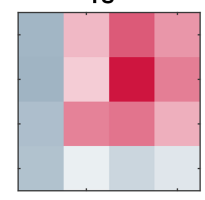

(e) $\mathrm{PI}_{\mathrm{TS}}$

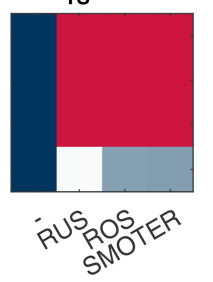

(c) $\mathrm{CE}_{\mathrm{HS}}$

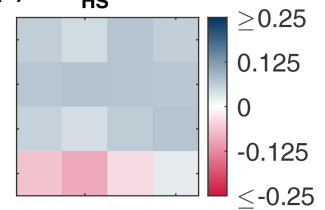

(f) $\mathrm{PI}_{\mathrm{HS}}$

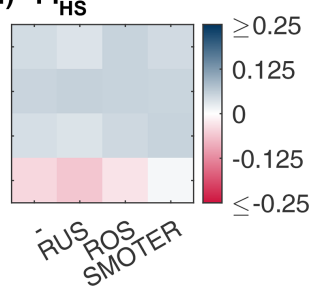

Figure 10. Change in (absolute) performance of CE (a), $\mathrm{CE}_{\mathrm{TS}}(\mathbf{b})$, $\mathrm{CE}_{\mathrm{HS}}(\mathbf{c}), \mathrm{PI}(\mathbf{d}), \mathrm{PI}_{\mathrm{TS}}(\mathbf{e})$, and $\mathrm{PI}_{\mathrm{HS}}$ (f) produced by combinations of resampling (listed along the $x$ axis) and ensemble (listed along the $y$ axis) methods for the Don River models.

of the Don River TFs (see Fig. 4), and thus the sum of the squared error between the naive model and observed stage is also low, reducing the PI value. The low value of $\mathrm{PI}_{\mathrm{TS}}$ is attributed to the quality of the naive model, not the inaccuracy of the ANN counterpart. Note that $\mathrm{PI}_{\mathrm{HS}}$ are typically slightly higher than the overall PI: during high stage, there is greater variability; thus, the naive model is less accurate, resulting in a higher PI score.

\subsection{Comparison of resampling and ensemble methods}

This section provides a detailed comparison of performance across the different resampling and ensemble methods. As expected, all three resampling methods (RUS, ROS, and SMOTER) typically increase HS performance, often at the expense of TS performance. Based on results shown in Table 5, the SMOTER variations provide the highest performance for HS for the Bow River. SMOTER-RWB $\mathrm{CE}_{\mathrm{HS}}$ is 0.72 , an increase from 0.617 of the individual learner, whereas the SMOTER-Bagging $\mathrm{PI}_{\mathrm{HS}}$ is 0.144 , compared to -0.175 for the individual learner. These indicators suggest that the HS prediction accuracy has improved slightly using these SMOTER variations. The results shown in Table 6 for the Don River indicate that the best improvements for HS prediction accuracy are provided by the RUS-Bagging method: the $\mathrm{CE}_{\mathrm{HS}}$ is 0.585 (an increase from 0.511 of the individual learner), and the $\mathrm{PI}_{\mathrm{HS}}$ is 0.668 (an increase from 0.61 of the individual learner). While both these metrics show an improvement in HS prediction accuracy for the Don River, the improvements are relatively small compared to the performance improvement for the Bow River. ROS often exhibits poorer performance than SMOTER and RUS. Previous research has noted the tendency for ROS-based models to overfit, due to the high number of duplicate samples (Yap et al., 2014). RUS, despite using considerably fewer training 
data for each individual learner, is not as prone to overfitting as ROS. The RUS-Bagging models consistently outperform the RUS-RWB models; this may be due to the repeated resampling; thus, RUS-Bagging uses many more of the original training samples, while RUS-RWB only uses $20 \%$ of the original data.

Figures 9 and 10 show absolute changes in CE and PI relative to the individual learner for the Bow and Don rivers, respectively, for the entire dataset, the TS, and the HS. Performance is colourised in a 2D matrix to facilitate comparisons in performance between each resampling method across ensemble types and vice versa. From these figures, it is apparent that SMOTER generally produces the largest improvements in HS performance, for both CE and PI and for both watersheds. The SMOTER methods are also generally the least detrimental to TS performance for both watersheds, as compared to ROS and RUS. Notably, SMOTER is the only resampling method whose performance does not decrease when used in combination with LSBoost. However, the change in performance due to SMOTER is marginal compared to the models without resampling. For the Bow River, the largest improvements between the best models with no resampling and the best models with resampling for $\mathrm{CE}_{\mathrm{HS}}$ and $\mathrm{PI}_{\mathrm{HS}}$ are 0.001 and 0.016 , respectively. For the Don River, the same improvements are 0.004 and 0.005 , respectively. The remaining resampling methods (RUS and ROS) also generally tend to improve HS performance across the ensemble techniques; however, this improvement is not consistent, as is the case with SMOTER, and the decrease in TS performance is also higher. Thus, while SMOTER provides consistent improvements over the non-resampling methods for CE and PI (entire, TS, and HS), RUS and ROS only provide minor improvements to HS performance.

When looking at the resampling methods, the RWB ensembles exhibit competitive performance compared to the other ensemble methods, despite their lower diversity. These ensembles represent a considerable improvement over the individual learner and often achieve higher performance compared to the other, more complex ensemble methods, as shown in Tables 5 and 6 . This suggests that using RWB is useful for improving CE and PI performance (for entire TS and HS) as compared to the single, individual learner. For the Bow River, the RWB ensembles improve the PI for each case (PI, $\mathrm{PI}_{\mathrm{TS}}$ and $\mathrm{PI}_{\mathrm{HS}}$ ) while only improving $\mathrm{CE}_{\mathrm{HS}}$. For the Don River models, a notable increase in performance is seen for both CE and PI (entire and HS datasets); however, when combined with the resampling techniques (RUS, ROS, and SMOTER), the TS performance metrics exhibit poorer performance.

The bagging ensembles also perform well, typically outperforming the RWB counterparts and following the same trends described above. This is likely due to their repeated resampling, which achieves greater ensemble diversity compared to the RWB models, for which resampling only occurs once. This result is consistent with a previous compar- ison of bagging and boosting (Shu and Burn, 2004). Like RWB and bagging, AdaBoost improves model performance compared to the individual learner but is typically slightly poorer compared to RWB and bagging and has higher variability in terms of improvement to model performance across all model types and both watersheds. The RWB, bagging, and Adaboost models consistently improve TS and HS performance compared to the individual learner regardless of whether they are combined with a resampling strategy.

The LSBoost models have the poorest HS performance out of all the ensemble methods studied. This is consistent across all resampling methods and both watersheds. In contrast, the change in performance for $\mathrm{CE}_{\mathrm{TS}}$ and $\mathrm{PI}_{\mathrm{TS}}$ is less detrimental when using LSBoost, suggesting that this method is not wellsuited to improving HS performance. The LSBoost models are slightly overfitted despite utilising the stop training for calibrating the ANN ensemble members. This is indicated by the degradation in performance between the calibration and test datasets, a change which is larger than that seen in the other ensemble models. This is most noticeable for the RUSLSBoost models for both the Bow and the Don rivers, which are more prone to overfitting compared to other models, due to the smaller number of training samples. The $\mathrm{CE}$ decreases from 0.97 to 0.902 for the Bow River and from 0.835 to 0.715 for the Don River; none of the other models that use RUS exhibits such a gap between train and test performance.

The overfitting produced by the boosting methods is consistent with previous research, which finds that boosting is sometimes prone to overfitting on real-world datasets (Vezhnevets and Barinova, 2007). One reason that the improvements made by the boosting methods (AdaBoost and LSBoost) are not more substantial may be the use of ANNs as individual learners. ANNs typically have more degrees of freedom compared to the decision trees that are most commonly used as individual learners; thus, the additional complexity offered by boosting does little to improve model predictions. Additionally, the boosting methods further increase the effective degrees of freedom of the predictions. Nevertheless, these methods still tend to improve performance over that of the individual learner. Ensembles of less complex models such as regression trees are expected to produce relatively larger improvements when relative to the single model predictions.

As discussed in Sect. 2.1, a fixed threshold is used to distinguish between high and typical stage values, which was set to $80 \%$ for the results presented above. Figure 11 shows the effects of the fixed threshold increasing from the 50th to 90th percentiles of the stage distribution. These plots show the relative effects of SMOTER-Bagging compared to simple bagging; these configurations were selected for this comparison since they both exhibited relatively good, consistent performance. A performance ratio greater than 1 indicates that the SMOTER-Bagging model has greater error compared to the bagging model; 1 indicates that they have the same performance, and, at less than 1, improved performance. Error is 


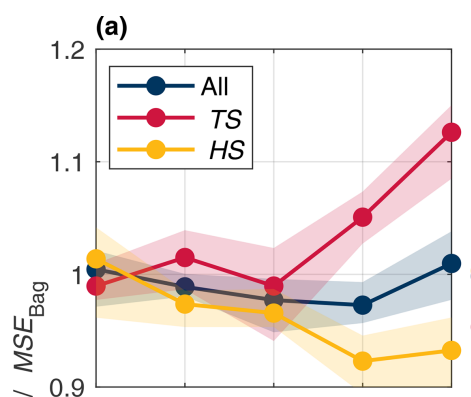

(c)
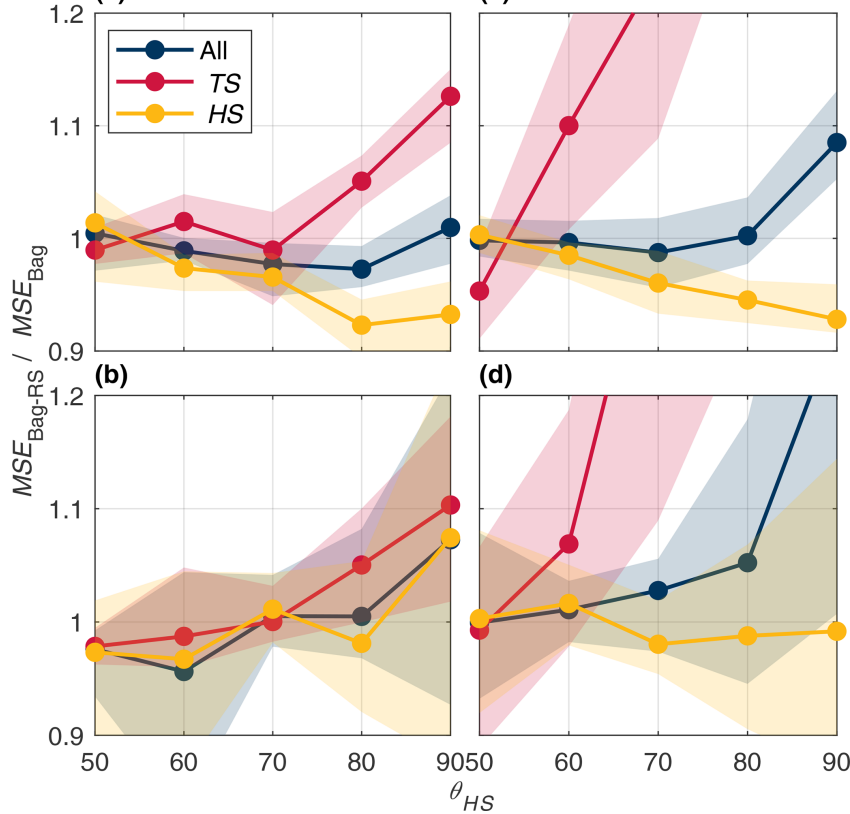

(d)

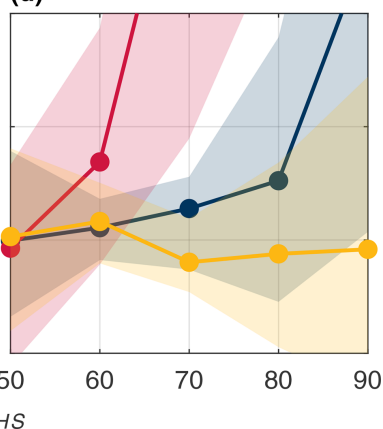

Figure 11. MSE ratio between the bagging and SMOTER-Bagging models for the Bow River calibration (a), Bow test (c), Don River calibration (b), and Don test (d) partitions across high stage threshold values ranging from the 50th to 90th percentile stage values.

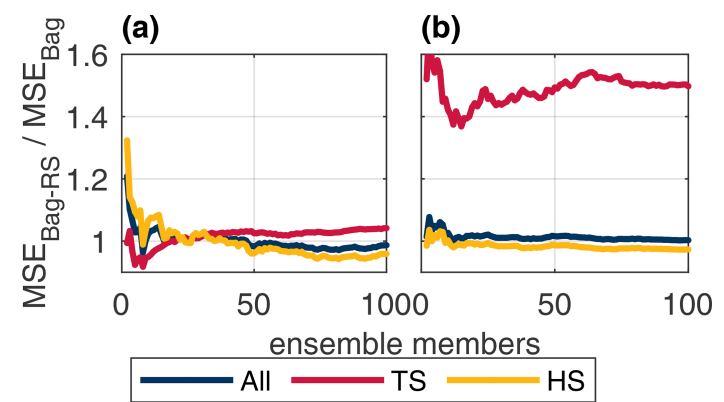

Figure 12. Test MSE ratio between the bagging and SMOTERBagging models for the Bow (a) and Don (b) rivers across ensemble size.

presented for all stage values as well as the TS and HS subsets. The calibration plots illustrate an asymmetric trade-off between HS and TS error. For a given $\theta_{\mathrm{HS}}$ value, the error ratio of the TS subset increases more than the decline in HS error. More importantly, the improvements in HS performance obtained in calibration are considerably less pronounced in the test dataset, despite a loss in TS performance.

Figure 12 illustrates the effects of varying the ensemble size, i.e. number of resampling repetitions, for the SMOTERBagging model relative to the simple bagging model. The plot shows the relative improvement in HS produced by the SMOTER resampling as the ensemble size increases, reaching a steady value at an ensemble size of approximately 70 for both the Don and Bow systems. This is larger than that re-

quired for the simple bagging model to reach steady performance, shown in Fig. 6, indicating that SMOTER requires more resampling than regular resampling with replacement (default in bagging) in order to reach stable performance. Consistent with observations made from Fig. 11, an asymmetric trade-off between typical and high stage performance is noted, illustrated by a disproportionate increase in error on the typical stage relative to the improvement on the high stage.

\subsection{Limitations and future work}

A limitation of this study is the lack of a systematic caseby-case hyperparameter optimisation of the models. The individual learner parameters (e.g. topology, activation function) were constant across all ensemble members. Likewise, the ensemble hyperparameters were not optimised but simply tuned using an ad hoc approach. A systematic approach to hyperparameter optimisation for each model will likely yield improved model performance. However, hyperparameter optimisation on such a scale would be very computationally expensive. Similarly, the selection of the HS threshold may affect $\mathrm{CE}_{\mathrm{HS}}$ and $\mathrm{PI}_{\mathrm{HS}}$ performance, and the sensitivity of the model performance of this threshold should be explored. This research featured resampling and ensemble methods for improving prediction accuracy across an imbalanced target dataset, i.e. the high stage. In addition to imbalanced target data, flood-forecasting applications commonly have imbalanced cost; for example, underprediction is typically more costly than overprediction. The use of cost functions, such as asymmetric weighting applied to underpredictions and overpredictions, for flood forecasting has been shown to reduce underprediction of flooding Toth, 2016. Many cost-sensitive ensemble techniques (e.g. Galar et al., 2012) have yet to be explored in the context of flood-forecasting models and should be the focus of future work.

\section{Conclusion}

This research presented the first systematic comparison of the effects of combined resampling and ensemble techniques for improving the accuracy of flow-forecasting models, specifically for high stage (infrequent) observations. Methods were applied to two Canadian watersheds, the Bow River in Alberta and the Don River in Ontario. This research attempts to address the widespread problem of poor performance on high stage when using data-driven approaches such as ANNs. Improving performance on high stage is essential for model applications such as early flood warning systems. Three resampling and four ensemble techniques are implemented as part of ANN flow-forecasting models for both watersheds. These methods are assessed independently and systematically combined in hybrid approaches so as to assess their efficacy for improving high stage performance. A major contribution of 
this paper is the comprehensive evaluation of these hybrid methods, most of which are the first instances in the water resource field. While methodologies for these combination methods are available in the existing machine-learning literature, our proposed implementation of SMOTER-AdaBoost is a novel improvement. Results demonstrate that resampling methods, when embedded in ensemble algorithms, generally only produce a small improvement in high stage performance, based on CE and PI; the SMOTER variation provided the most consistent improvements. An asymmetric trade-off between typical and high stage performance is observed in which improved high stage performance produced disproportionately worse typical flow performance. Such a tradeoff should be carefully considered while implementing these methods. Further research on this topic may explore the combination of cost-sensitive approaches with ensemble methods, which would allow for more aggressive penalisation of poor accuracy on high stage. 


\section{Appendix A: Input variable selection results}

Table A1. List of the 25 most useful inputs identified using the PC IVS algorithm for the Bow and Don River watersheds, selected from the set of candidate inputs. Input variables are encoded in the following format: "station ID"_"variable" "statistic" "lagged timesteps". Variable abbreviations "WL" and "Precip" refer to water level (stage) and precipitation.

\begin{tabular}{lll}
\hline rank & Bow & Don \\
\hline 1 & 05BH004 WL Mean L4 & HY022 WL Mean L4 \\
2 & 05BB001 WL Max L4 & HY008 Precip Sum L4 \\
3 & 05BB001 WL Min L12 & HY019 WL Mean L4 \\
4 & 05BH004 WL Mean L5 & HY008 Precip Sum L5 \\
5 & Calgary Temp Max L4 & HY027 Precip Sum L4 \\
6 & 05BB001 WL Max L6 & HY017 WL Mean L4 \\
7 & 05BH004 WL Mean L15 & HY022 WL Mean L5 \\
8 & Calgary Precip Sum L5 & HY008 Precip Sum L8 \\
9 & Calgary Temp Min L10 & HY027 Precip Sum L6 \\
10 & Calgary Precip Sum L11 & HY017 WL Mean L5 \\
11 & 05BH004 WL Max L4 & HY027 Precip Sum L5 \\
12 & 05BH004 WL Min L4 & HY008 Precip Sum L10 \\
13 & 05BH004 WL Max L7 & HY019 WL Mean L7 \\
14 & Calgary Precip Sum L7 & HY080 WL Mean L4 \\
15 & 05BB001 WL Min L15 & HY008 Precip Sum L11 \\
16 & 05BH004 WL Min L8 & HY008 Precip Sum L6 \\
17 & Calgary Precip Sum L10 & HY080 WL Mean L6 \\
18 & 05BH004 WL Max L12 & HY027 Precip Sum L7 \\
19 & Calgary Precip Sum L6 & HY022 WL Mean L6 \\
20 & 05BB001 WL Max L5 & HY027 Precip Sum L8 \\
21 & Calgary Temp Min L15 & HY022 WL Mean L7 \\
22 & 05BH004 WL Min L6 & HY080 WL Mean L5 \\
23 & 05BH004 WL Mean L6 & HY017 WL Mean L6 \\
24 & 05BH004 WL Max L5 & HY080 WL Mean L7 \\
25 & 05BB001 WL Min L9 & HY019 WL Mean L6 \\
\hline & &
\end{tabular}

\section{Appendix B: Pseudocode}

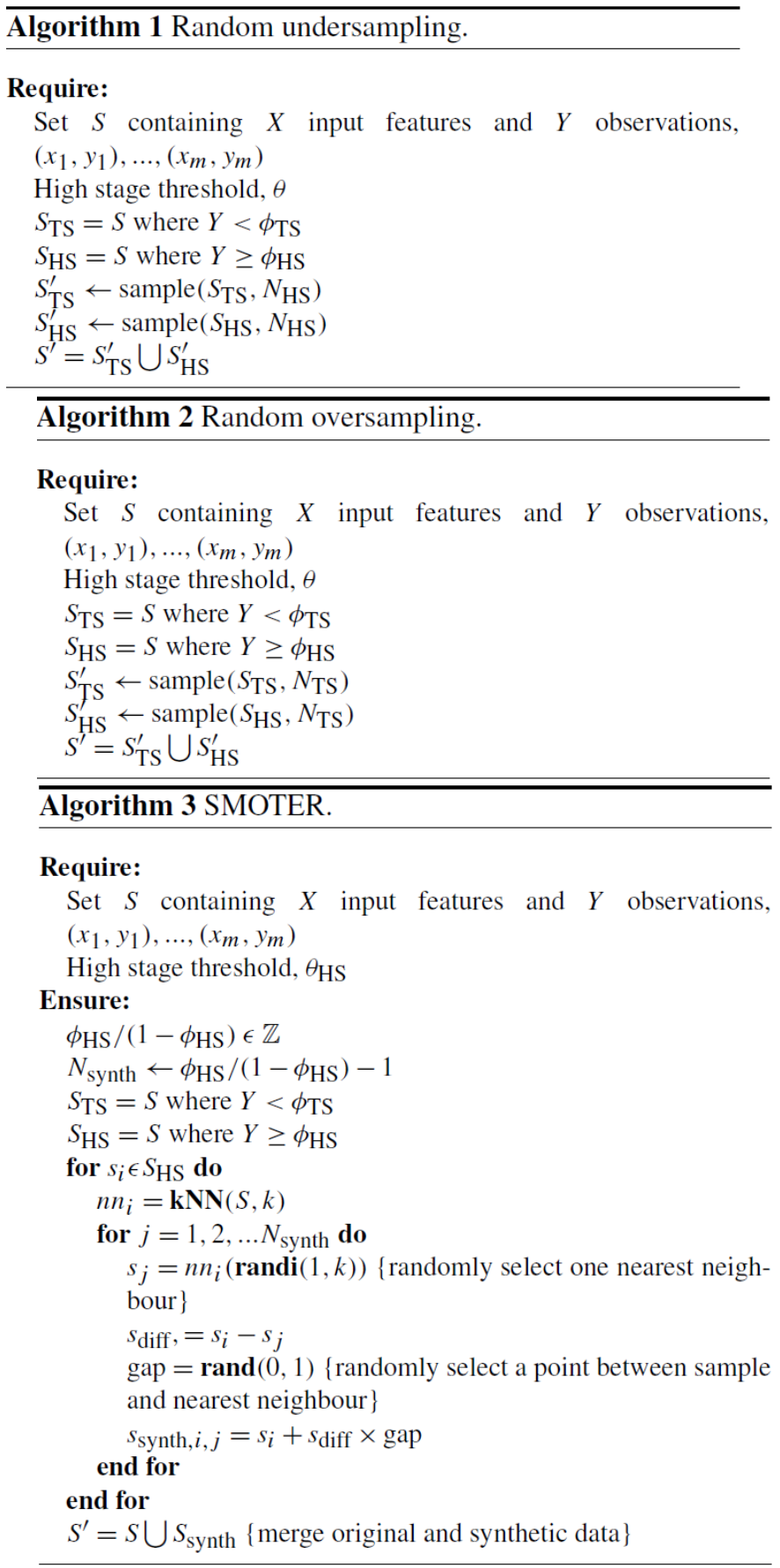




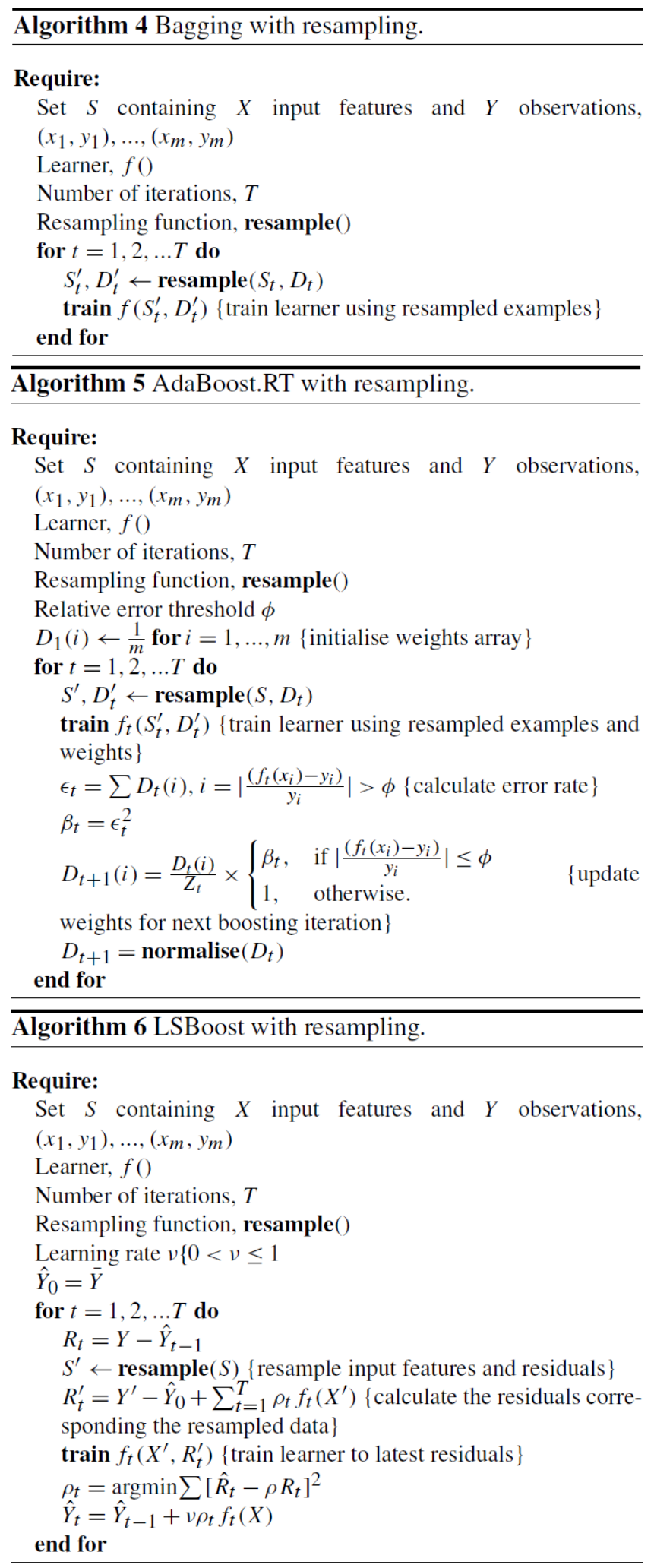


Data availability. The authors cannot redistribute the data used in this research, which must be obtained by request to the respective organisation. The temporal data used in this research may be obtained from the City of Calgary (Bow River precipitation and temperature), the Toronto and Region Conservation Authority (Don River precipitation and stage), Environment Canada (Don River temperature), and the Water Survey of Canada (Bow River stage). Figure 1 was produced using data from the following sources: Esri (aerial basemap Esri, 2020), DMTI Spatial Inc. accessed via Scholars GeoPortal (surface water and Bow River watershed boundary DMTI Spatial Inc., 2014a, b, c, 2019), and the TRCA (Don River watershed boundary Toronto and Region Conservation Authority, 2020b). Monitoring station locations were obtained from the metadata for the respective temporal datasets.

Author contributions. ES conducted the conceptualisation, formal analysis, investigation, method development, visualisation, and original draft. ES, KA, and UTK all contributed to writing and review. Funding acquisition and supervision were performed by UTK.

Competing interests. The authors declare that they have no known competing financial interests or personal relationships that could have appeared to influence the work reported in this paper.

Disclaimer. The views expressed in this paper are those of the authors and do not necessarily reflect the views or policies of the affiliated organisations.

Acknowledgements. The authors would like to thank the City of Calgary, the Toronto and Region Conservation Authority, and Environment Canada for providing data used in this research.

Financial support. This research has been supported by the Natural Sciences and Engineering Research Council of Canada (grant no. RGPIN-2017-05661).

Review statement. This paper was edited by Elena Toth and reviewed by K.S. Kasiviswanathan and two anonymous referees.

\section{References}

Abbot, J. and Marohasy, J.: Input selection and optimisation for monthly rainfall forecasting in Queensland, Australia, using artificial neural networks, Atmos. Res., 138, 166-178, https://doi.org/10.1016/j.atmosres.2013.11.002, 2014.

Abrahart, R. J., Heppenstall, A. J., and See, L. M.: Timing error correction procedure applied to neural network rainfall-runoff modelling, Hydrolog. Sci. J., 52, 414-431, https://doi.org/10.1623/hysj.52.3.414, 2007.

Abrahart, R. J., Anctil, F., Coulibaly, P., Dawson, C. W., Mount, N. J., See, L. M., Shamseldin, A. Y., Solomatine,
D. P., Toth, E., and Wilby, R. L.: Two decades of anarchy? Emerging themes and outstanding challenges for neural network river forecasting, Prog. Phys. Geog., 36, 480-513, https://doi.org/10.1177/0309133312444943, 2012.

Alobaidi, M. H., Meguid, M. A., and Chebana, F.: Predicting seismic-induced liquefaction through ensemble learning frameworks, Sci. Rep.-UK, 9, 11786, https://doi.org/10.1038/s41598019-48044-0, 2019.

Anctil, F. and Lauzon, N.: Generalisation for neural networks through data sampling and training procedures, with applications to streamflow predictions, Hydrol. Earth Syst. Sci., 8, 940-958, https://doi.org/10.5194/hess-8-940-2004, 2004.

Atieh, M., Taylor, G., Sattar, A. M. A., and Gharabaghi, B.: Prediction of flow duration curves for ungauged basins, J. Hydrol., 545, 383-394, https://doi.org/10.1016/j.jhydrol.2016.12.048, 2017.

Banjac, G., Vašak, M., and Baotić, M.: Adaptable urban water demand prediction system, Water Supply, 15, 958-964, https://doi.org/10.2166/ws.2015.048, 2015.

Barzegar, R., Ghasri, M., Qi, Z., Quilty, J., and Adamowski, J.: Using bootstrap ELM and LSSVM models to estimate river ice thickness in the Mackenzie River Basin in the Northwest Territories, Canada, J. Hydrol., 577, 123903, https://doi.org/10.1016/j.jhydrol.2019.06.075, 2019.

Bennett, N. D., Croke, B. F., Guariso, G., Guillaume, J. H., Hamilton, S. H., Jakeman, A. J., Marsili-Libelli, S., Newham, L. T., Norton, J. P., Perrin, C., Pierce, S. A., Robson, B., Seppelt, R., Voinov, A. A., Fath, B. D., and Andreassian, V.: Characterising performance of environmental models, Environ. Modell. Softw., 40, 1-20, https://doi.org/10.1016/j.envsoft.2012.09.011, 2013.

Błaszczyński, J. and Stefanowski, J.: Neighbourhood sampling in bagging for imbalanced data, Neurocomputing, 150, 529-542, https://doi.org/10.1016/j.neucom.2014.07.064, 2015.

Breiman, L.: Bagging predictors, Mach. Learn., 24, 123-140, https://doi.org/10.1007/BF00058655, 1996.

Brown, G., Wyatt, J., Harris, R., and Yao, X.: Diversity creation methods: A survey and categorisation, Inform. Fusion, 6, 5-20, https://doi.org/10.1016/j.inffus.2004.04.004, 2005.

Cannon, A. J. and Whitfield, P. H.: Downscaling recent streamflow conditions in British Columbia, Canada using ensemble neural network models, J. Hydrol., 259, 136-151, https://doi.org/10.1016/S0022-1694(01)00581-9, 2002.

Chapi, K., Singh, V. P., Shirzadi, A., Shahabi, H., Bui, D. T., Pham, B. T., and Khosravi, K.: A novel hybrid artificial intelligence approach for flood susceptibility assessment, Environ. Modell. Softw., 95, 229-245, https://doi.org/10.1016/j.envsoft.2017.06.012, 2017.

Chawla, N. V., Bowyer, K. W., Hall, L. O., and Kegelmeyer, W. P.: SMOTE: Synthetic minority over-sampling technique, J. Artif. Intell. Res., 16, 321-357, https://doi.org/10.1613/jair.953, 2002.

Chen, W., Hong, H., Li, S., Shahabi, H., Wang, Y., Wang, X., and Ahmad, B. B.: Flood susceptibility modelling using novel hybrid approach of reduced-error pruning trees with bagging and random subspace ensembles, J. Hydrol., 575, 864-873, https://doi.org/10.1016/j.jhydrol.2019.05.089, 2019.

Crochemore, L., Perrin, C., Andréassian, V., Ehret, U., Seibert, S. P., Grimaldi, S., Gupta, H., and Paturel, J.-E.: Comparing expert judgement and numerical criteria for hydrograph evaluation, Hydrolog. Sci. J., 60, 402-423, https://doi.org/10.1080/02626667.2014.903331, 2015. 
Dawson, C. W. and Wilby, R. L.: Hydrological modelling using artificial neural networks, Prog. Phys. Geogr., 25, 80-108, https://doi.org/10.1177/030913330102500104, 2001.

de Vos, N. and Rientjes, T.: Correction of Timing Errors of Artificial Neural Network Rainfall-Runoff Models, in: Practical Hydroinformatics, pp. 101-112, Springer, Berlin, Heidelberg, https://doi.org/10.1007/978-3-540-79881-1_8, 2009.

de Vos, N. J. and Rientjes, T. H. M.: Constraints of artificial neural networks for rainfall-runoff modelling: trade-offs in hydrological state representation and model evaluation, Hydrol. Earth Syst. Sci., 9, 111-126, https://doi.org/10.5194/hess-9-111-2005, 2005.

Díez-Pastor, J. F., Rodríguez, J. J., García-Osorio, C., and Kuncheva, L. I.: Random Balance: Ensembles of variable priors classifiers for imbalanced data, Knowledge-Based Syst., 85, 96-111, https://doi.org/10.1016/j.knosys.2015.04.022, 2015a.

Díez-Pastor, J. F., Rodríguez, J. J., García-Osorio, C. I., and Kuncheva, L. I.: Diversity techniques improve the performance of the best imbalance learning ensembles, Inform. Sci., 325, 98 117, https://doi.org/10.1016/j.ins.2015.07.025, 2015 b.

DMTI Spatial Inc.: Major Water Regions (MJWTR), available at: http://geo.scholarsportal.info/\#r/details/_uri@=311685684 (last access: 12 May 2021), 2014a.

DMTI Spatial Inc.: Intermittent Water (MNINR), available at: http: //geo.scholarsportal.info/\#r/details/_uri@=2422157200 (last access: 12 May 2021), 2014b.

DMTI Spatial Inc.: Minor Water Regions (MNWTR), available at: http://geo.scholarsportal.info/\#r/details/_uri@=2840086328 (last access: 12 May 2021), 2014c.

DMTI Spatial Inc.: Watersheds Region, available at: http://geo. scholarsportal.info/\#r/details/_uri@=2751227225 (last access: 12 May 2021), 2019.

Duncan, A.: The analysis and application of Artificial Neural Networks for early warning systems in hydrology and the environment, $\mathrm{PhD}$ thesis, College of Engineering, Mathematics and Physical Sciences, University of Exeter, Exeter, UK, 2014

Ehret, U. and Zehe, E.: Series distance - an intuitive metric to quantify hydrograph similarity in terms of occurrence, amplitude and timing of hydrological events, Hydrol. Earth Syst. Sci., 15, 877 896, https://doi.org/10.5194/hess-15-877-2011, 2011.

Erdal, H. I. and Karakurt, O.: Advancing monthly streamflow prediction accuracy of CART models using ensemble learning paradigms, J. Hydrol., 477, 119-128, https://doi.org/10.1016/j.jhydrol.2012.11.015, 2013.

Esri: World Imagery, available at: https://services.arcgisonline. com/ArcGIS/rest/services/World_Imagery/MapServer (last access: 12 May 2021), 2020.

Fernando, T., Maier, H., and Dandy, G.: Selection of input variables for data driven models: An average shifted histogram partial mutual information estimator approach, J. Hydrol., 367, 165-176, https://doi.org/10.1016/j.jhydrol.2008.10.019, 2009.

Fleming, S. W., Bourdin, D. R., Campbell, D., Stull, R. B., and Gardner, T.: Development and operational testing of a superensemble artificial intelligence flood-forecast model for a pacific northwest river, J. Am. Water Resour. As., 51, 502-512, https://doi.org/10.1111/jawr.12259, 2015.

Freund, Y. and Schapire, R. E.: Experiments with a New Boosting Algorithm, in: ICML'96: Proceedings of the 13th International Conference on Machine Learning, Bari, Italy, 3-6 July 1996, 148-156, 1996.
Friedman, J. H.: Greedy Function Approximation: A Gradient Boosting Machine, Ann. Stat., 29, 1189-1232, 2000.

Galar, M., Fernandez, A., Barrenechea, E., Bustince, H., and Herrera, F.: A review on ensembles for the class imbalance problem: Bagging-, boosting-, and hybrid-based approaches, IEEE T. Syst. Man Cy. C, 42, 463-484, https://doi.org/10.1109/TSMCC.2011.2161285, 2012.

Galar, M., Fernández, A., Barrenechea, E., and Herrera, F.: EUSBoost: Enhancing ensembles for highly imbalanced data-sets by evolutionary undersampling, Pattern Recognition, 46, 3460 3471, https://doi.org/10.1016/j.patcog.2013.05.006, 2013.

Govindaraju, R. S.: Artificial Neural Networks in Hydrology. II: Hydrologic Applications, J. Hydrol. Eng., 5, 124137, https://doi.org/10.1061/(ASCE)1084-0699(2000)5:2(124), 2000.

Gupta, H. V., Kling, H., Yilmaz, K. K., and Martinez, G. F.: Decomposition of the mean squared error and NSE performance criteria: Implications for improving hydrological modelling, J. Hydrol., 377, 80-91, https://doi.org/10.1016/j.jhydrol.2009.08.003, 2009.

Haixiang, G., Yijing, L., Shang, J., Mingyun, G., Yuanyue, H., and Bing, G.: Learning from class-imbalanced data: Review of methods and applications, Expert Syst. Appl., 73, 220-239, https://doi.org/10.1016/j.eswa.2016.12.035, 2017.

Hastie, T., Tibshirani, R., and Friedman, J.: Elements of Statistical Learning, 2nd ed., no. 2 in Springer Series in Statistics, Springer New York, New York, NY, https://doi.org/10.1007/978-0-38784858-7, 2009.

He, J., Valeo, C., Chu, A., and Neumann, N. F.: Prediction of eventbased stormwater runoff quantity and quality by ANNs developed using PMI-based input selection, J. Hydrol., 400, 10-23, https://doi.org/10.1016/j.jhydrol.2011.01.024, 2011.

Khan, U. T., He, J., and Valeo, C.: River flood prediction using fuzzy neural networks: an investigation on automated network architecture, Water Sci. Technol., 2017, 238-247, https://doi.org/10.2166/wst.2018.107, 2018.

Lauzon, N., Anctil, F., and Baxter, C. W.: Clustering of heterogeneous precipitation fields for the assessment and possible improvement of lumped neural network models for streamflow forecasts, Hydrol. Earth Syst. Sci., 10, 485-494, https://doi.org/10.5194/hess-10-485-2006, 2006.

Li, J., Zhang, C., Zhang, X., He, H., Liu, W., and Chen, C.: Temperature Compensation of Piezo-Resistive Pressure Sensor Utilizing Ensemble AMPSO-SVR Based on Improved Adaboost.RT, IEEE Access, 8, 12413-12425, https://doi.org/10.1109/ACCESS.2020.2965150, 2020.

Liu, S., Xu, J., Zhao, J., Xie, X., and Zhang, W.: Efficiency enhancement of a process-based rainfall-runoff model using a new modified AdaBoost.RT technique, Appl. Soft Comput., 23, 521529, https://doi.org/10.1016/j.asoc.2014.05.033, 2014.

López, V., Fernández, A., García, S., Palade, V., and Herrera, F.: An insight into classification with imbalanced data: Empirical results and current trends on using data intrinsic characteristics, Inform. Sciences, 250, 113-141, https://doi.org/10.1016/j.ins.2013.07.007, 2013.

Mahamud, K. R. K., Zorkeflee, M., and Din, A. M.: Fuzzy Distancebased Undersampling Technique for Imbalanced Flood Data, in: Proceedings of the Knowledge Management International Conference, Chiang Mai, Thailand, 29-30 August 2016, 2016. 
Maier, H. R. and Dandy, G. C.: Neural networks for the prediction and forecasting of water resources variables: A review of modelling issues and applications, Environ. Modell. Softw., 15, 101124, https://doi.org/10.1016/S1364-8152(99)00007-9, 2000.

Maier, H. R., Jain, A., Dandy, G. C., and Sudheer, K.: Methods used for the development of neural networks for the prediction of water resource variables in river systems: Current status and future directions, Environ. Modell. Softw., 25, 891-909, https://doi.org/10.1016/j.envsoft.2010.02.003, 2010.

Moniz, N., Branco, P., and Torgo, L.: Resampling strategies for imbalanced time series forecasting, International Journal of Data Science and Analytics, 3, 161-181, https://doi.org/10.1007/s41060-017-0044-3, 2017a.

Moniz, N., Branco, P., and Torgo, L.: Evaluation of Ensemble Methods in Imbalanced Regression Tasks, in: Proceedings of the First International Workshop on Learning with Imbalanced Domains: Theory and Applications, Skopje, Macedonia, 22 September 2017, 129-140, 2017bb.

Moniz, N., Ribeiro, R., Cerqueira, V., and Chawla, N.: SMOTEBoost for Regression: Improving the Prediction of Extreme Values, in: 2018 IEEE 5th International Conference on Data Science and Advanced Analytics (DSAA), 150-159, IEEE, https://doi.org/10.1109/DSAA.2018.00025, 2018.

Mosavi, A., Ozturk, P., and Chau, K.-w.: Flood Prediction Using Machine Learning Models: Literature Review, Water, 10, 1536, https://doi.org/10.3390/w10111536, 2018.

Ni, L., Wang, D., Wu, J., Wang, Y., Tao, Y., Zhang, J., and Liu, J.: Streamflow forecasting using extreme gradient boosting model coupled with Gaussian mixture model, J. Hydrol., 586, 124901, https://doi.org/10.1016/j.jhydrol.2020.124901, 2020.

Nirupama, N., Armenakis, C., and Montpetit, M.: Is flooding in Toronto a concern?, Nat. Hazards, 72, 1259-1264, https://doi.org/10.1007/s11069-014-1054-2, 2014.

Ouarda, T. B. M. J. and Shu, C.: Regional low-flow frequency analysis using single and ensemble artificial neural networks, Water Resour. Res., 45, W11428, https://doi.org/10.1029/2008WR007196, 2009.

Papacharalampous, G., Tyralis, H., Langousis, A., Jayawardena, A. W., Sivakumar, B., Mamassis, N., Montanari, A., and Koutsoyiannis, D.: Probabilistic hydrological postprocessing at scale: Why and how to apply machinelearning quantile regression algorithms, Water, 11, 2126, https://doi.org/10.3390/w11102126, 2019.

Pisa, I., Santín, I., Vicario, J. L., Morell, A., and Vilanova, R.: Data preprocessing for ANN-based industrial time-series forecasting with imbalanced data, in: European Signal Processing Conference, 2019, European Signal Processing Conference, EUSIPCO, https://doi.org/10.23919/EUSIPCO.2019.8902682, 2019.

Razali, N., Ismail, S., and Mustapha, A.: Machine learning approach for flood risks prediction, IAES International Journal of Artificial Intelligence, 9, 73-80, https://doi.org/10.11591/ijai.v9.i1.pp73$80,2020$.

Saffarpour, S., Erechtchoukova, M. G., Khaiter, P. A., Chen, S. Y., and Heralall, M.: Short-term prediction of flood events in a small urbanized watershed using multi-year hydrological records, in: Proceedings of the 21st International Congress on Modelling and Simulation (MODSIM2015), Broadbeach, Australia, 29 November-4 December 2014, 2234-2240, https://doi.org/10.36334/MODSIM.2015.L7.saffarpour, 2015.
Seibert, S. P., Ehret, U., and Zehe, E.: Disentangling timing and amplitude errors in streamflow simulations, Hydrol. Earth Syst. Sci., 20, 3745-3763, https://doi.org/10.5194/hess-20-3745-2016, 2016.

Seiffert, C., Khoshgoftaar, T. M., Van Hulse, J., and Napolitano, A.: Resampling or reweighting: A comparison of boosting implementations, in: Proceedings - International Conference on Tools with Artificial Intelligence, ICTAI, 1, 445-451, https://doi.org/10.1109/ICTAI.2008.59, 2008.

Sharkey, A. J. C.: On Combining Artificial Neural Nets, Connection Science, 8, 299-314, https://doi.org/10.1080/095400996116785, 1996.

Sharma, A.: Seasonal to interannual rainfall probabilistic forecasts for improved water supply management: Part 1 - A strategy for system predictor identification, J. Hydrol., 239, 232-239, https://doi.org/10.1016/S0022-1694(00)00346-2, 2000.

Shrestha, D. L. and Solomatine, D. P.: Experiments with AdaBoost.RT, an improved boosting scheme for regression, Neural Computat., 18, 1678-1710, https://doi.org/10.1162/neco.2006.18.7.1678, 2006.

Shu, C. and Burn, D. H.: Artificial neural network ensembles and their application in pooled flood frequency analysis, Water Resour. Res., 40, W09301, https://doi.org/10.1029/2003WR002816, 2004.

Shu, C. and Ouarda, T. B.: Flood frequency analysis at ungauged sites using artificial neural networks in canonical correlation analysis physiographic space, Water Resour. Res., 43, W07438, https://doi.org/10.1029/2006WR005142, 2007.

Snieder, E., Shakir, R., and Khan, U.: A comprehensive comparison of four input variable selection methods for artificial neural network flow forecasting models, J. Hydrol., 583, 124299, https://doi.org/10.1016/j.jhydrol.2019.124299, 2020.

Solomatine, D. P. and Ostfeld, A.: Data-driven modelling: some past experiences and new approaches, J. Hydroinform., 10, 3 22, https://doi.org/10.2166/hydro.2008.015, 2008.

Solomatine, D. P. and Shrestha, D. L.: AdaBoost.RT: A boosting algorithm for regression problems, in: IEEE International Conference on Neural Networks - Conference Proceedings, 2, 1163 1168, https://doi.org/10.1109/ijcnn.2004.1380102, 2004.

Sudheer, K. P., Nayak, P. C., and Ramasastri, K. S.: Improving peak flow estimates in artificial neural network river flow models, Hydrol. Process., 17, 677-686, https://doi.org/10.1002/hyp.5103, 2003.

Sufi Karimi, H., Natarajan, B., Ramsey, C. L., Henson, J., Tedder, J. L., and Kemper, E.: Comparison of learning-based wastewater flow prediction methodologies for smart sewer management, J. Hydrol., 577, 123977, https://doi.org/10.1016/j.jhydrol.2019.123977, 2019.

Tiwari, M. K. and Chatterjee, C.: Uncertainty assessment and ensemble flood forecasting using bootstrap based artificial neural networks (BANNs), J. Hydrol., 382, 20-33, https://doi.org/10.1016/j.jhydrol.2009.12.013, 2010.

Tongal, H. and Booij, M. J.: Simulation and forecasting of streamflows using machine learning models coupled with base flow separation, J. Hydrol., 564, 266-282, https://doi.org/10.1016/j.jhydrol.2018.07.004, 2018.

Torgo, L., Ribeiro, R. P., Pfahringer, B., and Branco, P.: SMOTE for regression, in: Lecture Notes in Computer Science (including subseries Lecture Notes in Artificial Intelligence 
and Lecture Notes in Bioinformatics), 8154 LNAI, 378-389, https://doi.org/10.1007/978-3-642-40669-0_33, 2013.

Toronto and Region Conservation Authority: Lower Don River West Remedial Flood Protection Project, available at: https://trca.ca/conservation/green-infrastructure/ lower-don-river-west-remedial-flood-protection-project/ (last access: 12 May 2021), 2020a.

Toronto and Region Conservation Authority: Watersheds TRCA, available at: https://data.trca.ca/dataset/watersheds-trca (last access: 12 May 2021), 2020b.

Toth, E.: Estimation of flood warning runoff thresholds in ungauged basins with asymmetric error functions, Hydrol. Earth Syst. Sci., 20, 2383-2394, https://doi.org/10.5194/hess-20-23832016, 2016.

Vezhnevets, A. and Barinova, O.: Avoiding Boosting Overfitting by Removing Confusing Samples, in: Machine Learning: ECML 2007, 4701 LNAI, 430-441, Springer Berlin Heidelberg, Berlin, Heidelberg, https://doi.org/10.1007/978-3-54074958-5_40, 2007.

Wang, R., Zhang, X., and Li, M. H.: Predicting bioretention pollutant removal efficiency with design features: A data-driven approach, J. Environ. Manage., 242, 403-414, https://doi.org/10.1016/j.jenvman.2019.04.064, 2019a.

Wang, S.-H., Li, H.-F., Zhang, Y.-J., and Zou, Z.-S.: A Hybrid Ensemble Model Based on ELM and Improved AdaBoost.RT Algorithm for Predicting the Iron Ore Sintering Characters, Comput. Intel. Neurosc., 2019, 1-11, https://doi.org/10.1155/2019/4164296, 2019b.

Wang, W., Gelder, P. H., Vrijling, J. K., and Ma, J.: Forecasting daily streamflow using hybrid ANN models, J. Hydrol., 324, 383-399, https://doi.org/10.1016/j.jhydrol.2005.09.032, 2006.
Worland, S. C., Farmer, W. H., and Kiang, J. E.: Improving predictions of hydrological low-flow indices in ungaged basins using machine learning, Environ. Modell. Softw., 101, 169-182, https://doi.org/10.1016/j.envsoft.2017.12.021, 2018.

Wu, Y., Ding, Y., and Feng, J.: SMOTE-Boost-based sparse Bayesian model for flood prediction, Eurasip J. Wirel. Comm. 2020, 78, https://doi.org/10.1186/s13638-020-01689-2, 2020.

Yap, B. W., Rani, K. A., Rahman, H. A. A., Fong, S., Khairudin, Z., and Abdullah, N. N.: An Application of Oversampling, Undersampling, Bagging and Boosting in Handling Imbalanced Datasets, Lect. Notes Electr. Engr., 285 LNEE, 13-22, https://doi.org/10.1007/978-981-4585-18-7_2, 2014.

Zhan, C., Han, J., Zou, L., Sun, F., and Wang, T.: Heteroscedastic and symmetric efficiency for hydrological model evaluation criteria, Hydrol. Res., 50, 1189-1201, https://doi.org/10.2166/nh.2019.121, 2019.

Zhang, H., Yang, Q., Shao, J., and Wang, G.: Dynamic Streamflow Simulation via Online GradientBoosted Regression Tree, J. Hydrol. Eng., 24, 04019041, https://doi.org/10.1061/(ASCE)HE.1943-5584.0001822, 2019.

Zhang, Z.-L., Luo, X.-G., Yu, Y., Yuan, B.-W., and Tang, J.-F.: Integration of an improved dynamic ensemble selection approach to enhance one-vs-one scheme, Eng. Appl. Artif. Intel., 74, 43-53, https://doi.org/10.1016/j.engappai.2018.06.002, 2018.

Zhaowei, Q., Haitao, L., Zhihui, L., and Tao, Z.: Short-Term Traffic Flow Forecasting Method With M-B-LSTM Hybrid Network, IEEE Transactions on Intelligent Transportation Systems, 1-11, https://doi.org/10.1109/TITS.2020.3009725, 2020. 